\title{
Investigating Explosion Source Energy Partitioning and $L g$-Wave Excitation Using a Finite-Difference plus Slowness Analysis Method
}

\author{
by Xiao-Bi Xie, Zengxi Ge, and Thorne Lay
}

\begin{abstract}
A finite-difference modeling plus slowness analysis method is developed to investigate near-source explosion energy partitioning and $L g$-wave excitation. The finite-difference method is used to calculate seismic wave excitation and propagation, and an embedded array slowness analysis is used for quantifying how energy will be partitioned into the long-range propagation regime. Because of its high efficiency, the method can simulate near-source processes using very fine structures. A large number of source and model parameters can be examined for broadfrequency ranges. As examples, $P$ - $p S$-to- $L g$ and $S^{*}$-to- $L g$ conversions in the presence of near-source scattering are tested as mechanisms for $L g$-wave excitation. The numerical results reveal that the depth of the source and the depth of the scattering process have strong effects on $P$-to- $S$ conversion and partitioning of energy into trapped or leaking signals. The $L g$-wave excitation spectra from these mechanisms are also investigated. The modeling shows that $S^{*}$-to- $L g$ excitation is generally stronger for low frequencies and shallow source depths whereas $P$ - $p S$-to- $L g$ scattering is stronger for high frequencies.
\end{abstract}

\section{Introduction}

With the current emphasis on global monitoring for low-yield nuclear tests, regional seismic phases such as $L g$ have become very important for magnitude and yield estimation of underground nuclear tests (Nuttli, 1986; Xie, et al., 1996; Patton, 2001). In addition, various $P / S$-type amplitude ratios for high-frequency regional phases (e.g., $P n /$ $\mathrm{Sn}, \mathrm{Pn} / \mathrm{Lg}, \mathrm{Pg} / \mathrm{Lg}, \mathrm{Pg} / \mathrm{Sn}$ ) have become important for event discrimination (Taylor et al., 1989; Kim et al., 1993, 1997; Walter et al., 1995; Fisk et al., 1996; Taylor, 1996; Taylor and Hartse, 1997; Hartse et al., 1997; Fan and Lay, 1998a1998c; Xie, 2002; Bottone et al., 2002). The applications of regional phases for yield estimation and event discrimination are largely based on empirical approaches, and, although very promising in many cases, major questions exist about the nature of excitation of $S$-wave-dominated phases such as $L g$. There are similar questions regarding the relative excitation effects for $P / S$-type ratios in regional phases, in particular, given the huge scatter observed in both earthquake and explosion data populations.

There are now many observational and theoretical studies addressing the regional phase energy-partitioning issue in both the near-source environment and the propagationpath environment. Along the propagation path, the existence of small-scale heterogeneities in the crust and the associated seismic wave scattering has long been addressed by seismologists (Wu and Aki, 1988; Sato and Fehler, 1998), and its effect on the long-range energy partitioning has been doc- umented (Wu et al., 2000a, 2000b). In the source region, several possible near-source energy excitation mechanisms have been proposed, including $P$-to- $L g$ scattering, $p S$-to- $L g$ conversion at the free surface, $R g$-to- $L g$ coupling, $S^{*}$-to- $L g$ conversion, spall excitation of $S$, tectonic release, and rock damage (Gutowski et al., 1984; Lilwall, 1988; Day and Mclaughlin, 1991; Gupta et al., 1992, 1997; Wallace, 1991; Xie and Lay, 1994; Vogfjord, 1997; Johnson and Sammis, 2001).

Because of the complex excitation and energy-partitioning processes associated with regional phases, it is difficult to empirically separate the contribution of individual energypartitioning mechanisms by analysis of data. Numerical modeling approaches are thus of great importance for investigating the excitation and propagation of regional phases. Kennett and Mykkeltveit (1984) and Kennett (1989) used the coupled-mode method to calculate $L g$-wave propagation in crustal wave guides with weak lateral heterogeneities. Xie and Lay (1994) investigated $L g$-wave excitation using the full-wave finite-difference (FD) method. Jih (1995, 1996) investigated $R g$-to- $L g$ coupling as a possible $L g$ excitation mechanism. With an anelastic FD method, Bradley and Jones $(1998,1999)$ investigated $L g$ propagation and attenuation in Western China and India. Recently, using the 2D and 3D general Fourier methods, Bonner et al. (2003) investigated $R g$ and $L g$ generation and partially reproduced the observed spectrum from the Depth of Burial Experiment. 
Stevens et al. (2003) investigated the physical basis of explosion-generated $S$-waves using a 2D nonlinear FD method, which handles axisymmetric near-source effects, including spall, cracking, and nonlinear deformation.

The main disadvantages of these numerical methods are their low computation efficiency and huge computer memory requirement, especially when applied to investigate broadband $L g$ excitation. For the purpose of small nuclear test monitoring, the range of interest for $L g$-wave simulation involves a broad-frequency band $(0.2-10 \mathrm{~Hz})$ and long propagation distances (up to $1000 \mathrm{~km}$ or more). At the same time, factors that control the source energy partitioning depend on the detailed source mechanism and fine near-source velocity structure. In addition, multiple mechanisms may potentially contribute to the energy-partitioning process. Numerous parameters need to be tested to investigate the characteristics of these mechanisms, especially their contributions to the frequency-dependent features of observable discriminants. If random heterogeneities are to be considered, as is likely to be important for high-frequency signals, the results have to be calculated statistically from simulations using a large number of realizations. These factors limit the approach of complete FD synthesis for actual recording geometries.

Although there are continuing controversies about the dominant $P$-to- $S$ transfer mechanisms affecting regional phases, most investigators agree that appreciable energy from explosion sources is converted to $S$ waves in the nearsource region (Myers et al., 2003). The physical processes by which an explosion source generates regional phases can be described as energy partitioning taking place in the nearsource region. The partitioned energy subsequently propagates through a long wave guide, where secondary energypartitioning effects may occur, but these are less affected by the type of source involved. If the propagation effect is not of primary interest, it is desirable to avoid calculating the immensely time-consuming long-distance propagation part of the problem. To focus on the near-source energypartitioning processes, we developed a method based on the FD simulation and local slowness analysis. This method investigates energy partitioning right at the source region but quantifies how energy will transfer into the long-range propagation path, which is critical for comparisons with data. The localized analysis thereby isolates the physical processes controlling the energy partitioning.

In the following sections, we first present the method with numerical calculations used to demonstrate its validity. Then, by investigating two potential $L g$-wave excitation mechanisms, we demonstrate how this method can be used to investigate explosion source energy partitioning. This article does not provide a complete investigation of all $\mathrm{Lg}$ wave excitation mechanisms. Many important issues, e.g., the effects of free surface topography and dipping structures, $R g$-to- $L g$ conversion, and contributions from secondary sources, will be addressed in future work.

\section{Methodology}

We limit our 2D FD simulation to a relatively small model and analyze the wave field within the model to investigate the source energy partitioning and the excitation of trapped regional phases such as $L g$. Many authors (e.g., Frankel, 1989; Xie and Lay, 1994; Vogfjord, 1997) have pointed out that for $S$-wave energy to be trapped in the wave guide, reverberating to generate the $L g$-wave, it must propagate with postcritical angle at the Moho discontinuity. However, in the wave guide and especially in the nearsource region, the wave field is highly complex. It is impractical to trace each phase in the spatial-time domain. An alternate but equally valid way of tracking the wave energy is in the slowness domain. Multiply reflected waves may arrive simultaneously in time, but in the slowness domain their energy distribution gives clear information about the wave intensity, slowness, and propagation direction. Several methods can be used to transfer spatial-time domain data into slowness (or equivalently wavenumber) domain information, for example, frequency-wavenumber analysis or slant stacking. Here we use a local slant-stacking method to conduct slowness analysis, working simultaneously in both the space and slowness domains. We call this the finite-difference slowness analysis (FDSA) method.

\section{Local Slowness Analysis}

Two-dimensional local slant stacking in the horizontal and vertical directions can be expressed as:

$$
\begin{aligned}
\mathbf{u}(\mathbf{r}, \mathbf{p}, t, \omega)=\frac{1}{C_{1}} \sum_{r^{\prime}} W_{R}\left(\mathbf{r}^{\prime}-\mathbf{r}\right) \\
\mathbf{u}\left[\mathbf{r}^{\prime}, t-\mathbf{p} \cdot\left(\mathbf{r}^{\prime}-\mathbf{r}\right), \omega\right],
\end{aligned}
$$

where $\mathbf{r}=x \hat{\mathbf{e}}_{x}+z \hat{\mathbf{e}}_{z}$ is the $2 \mathrm{D}$ position vector, $\hat{\mathbf{e}}_{x}$ and $\hat{\mathbf{e}}_{z}$ are unit vectors in the $x$ and $z$ direction, $\mathbf{r}^{\prime}$ is the location of the receiver, $t$ is time, $\mathbf{u}(\mathbf{r}, t, \omega)$ is the bandpass-filtered synthetic seismogram with central frequency $\omega, W_{R}\left(\mathbf{r}^{\prime}-\mathbf{r}\right)$ is a twodimensional space window centered at $\mathbf{r}, \mathbf{p}=p \hat{\mathbf{e}}_{p}$ is the slowness vector, $\hat{\mathbf{e}}_{p}$ is the unit vector of the slowness direction, $p=v^{-1}$ is the wave slowness, $v=v_{p}$ or $v=v_{s}$ is $P$ or $S$-wave velocity, $C_{1}$ is a normalization factor determined by the size of the space window and bandwidth of the frequency filter. The space window is for a small vertical seismic array with size related to both space resolution and slowness resolution. A larger array gives better slowness resolution but tends to smear the spatial resolution, whereas a small array gives better spatial resolution but less accurate slowness calculation. A proper trade-off between space and slowness resolution is required. The receiver interval should be small enough to avoid spatial aliasing. The average energy density of the wave field as a function of space, time, slowness, and frequency can be obtained as 


$$
D(\mathbf{r}, \mathbf{p}, t, \omega)=\rho \overline{\dot{u}}^{2}(\mathbf{r}, \mathbf{p}, t, \omega),
$$

and the energy flux related to slowness vector $\mathbf{p}$ can be calculated as

$$
\mathbf{J}=v D(\mathbf{r}, \mathbf{p}, t, \omega) \hat{\mathbf{e}}_{p},
$$

where $\rho$ is the density and $\overline{\dot{u}}(\mathbf{r}, \mathbf{p}, t, \omega)$ is the root-meansquare (rms) amplitude of the stacked velocity seismogram $\dot{\mathbf{u}}(\mathbf{r}, \mathbf{p}, t, \omega)$. Similarly, the 1D horizontal slant stack can be calculated as

$$
\begin{aligned}
\mathbf{u}\left(\mathbf{r}, p_{x}, t, \omega\right)=\frac{1}{C_{2}} \sum_{x^{\prime}} W_{R}\left(x^{\prime}-x, z\right) \\
\mathbf{u}\left[x^{\prime}, z, t-p_{x}\left(x^{\prime}-x\right), \omega\right],
\end{aligned}
$$

where $p_{x}=p \hat{\mathbf{e}}_{p} \cdot \hat{\mathbf{e}}_{x}$ is the horizontal slowness, $W_{R}\left(x^{\prime}-x\right.$, $z$ ) is a $1 \mathrm{D}$ space window, and $C_{2}$ is a normalization factor similar to $C_{1}$ in equation (1). The average energy density as a function of $p_{x}$ can be expressed as

$$
D\left(\mathbf{r}, p_{x}, t, \omega\right)=\rho \overline{\dot{u}^{2}}\left(\mathbf{r}, p_{x}, t, \omega\right) .
$$

The pure horizontal energy flux related to horizontal slowness $p_{x}$ can be obtained from equation (3)

$$
J_{h}\left(\mathbf{r}, p_{x}, t, \omega\right)=\int \mathbf{J}(\mathbf{r}, \mathbf{p}, t, \omega) \cdot \hat{\mathbf{e}}_{x} d p_{z} .
$$

Alternatively, $J_{h}$ can be calculated from horizontal slant stacking, i.e., equations (4) and (5).

$$
J_{h}\left(\mathbf{r}, p_{x}, t, \omega\right)=v D\left(\mathbf{r}, p_{x}, t, \omega\right) \cdot \hat{\mathbf{e}}_{P} \cdot \hat{\mathbf{e}}_{x}
$$

where $\hat{\mathbf{e}}_{p} \cdot \hat{\mathbf{e}}_{x}=v p_{x}=\sin i$ and $i$ is the wave-incident angle (relative to the vertical direction). In the wave guide, the energy passing through a surface $S$ within a frequency range $\Omega$, a time window $T$, and a slowness band $P$ can be calculated as:

$$
E(S, P, T, \Omega)=\int_{S} \int_{P} \int_{T} \int_{\Omega} \mathbf{J} \cdot \hat{\mathbf{e}}_{n} d \omega d t d \mathbf{p} d s,
$$

where $\hat{\mathbf{e}}_{n}$ is the unit normal vector of the surface element $d s$, and $d \mathbf{p}=d p_{x} d p_{z}$. When choosing a vertical intersection as the surface, equation (8) becomes

$$
E(S, P, T, \Omega)=\int_{S} \int_{P} \int_{T} \int_{\Omega} J_{h} d \omega d t d p_{x} d z .
$$

Equations (8) and (9) provide the basis for extracting energy from joint domains. To investigate the near-source energy partitioning and regional phase excitation, the energy generated from specific mechanisms is decomposed into multiple domains and analyzed based on its dynamic and kine- matic properties. The condition for energy to be trapped in the crustal wave guide, i.e., $p_{x} \geq 1 / v_{S \text {-mantle }}$ (where $v_{S \text {-mantle }}$ is the upper-mantle $S$ velocity) is applied. We then use the joint window $(S, P, T, \Omega)$ to sort the trapped energy and estimate the contribution of specific mechanisms to regional phases such as $L g$. Equations (8) or (9) can be partially integrated, which allows the energy to be projected onto different domains. The analysis within multiple domains provides additional information to characterize the contributions from different mechanisms. This method has the flexibility that we can either intercept the entire wave-guide energy flux or just monitor the energy from specific phases or mechanisms. The calculation using $2 \mathrm{D}$ slowness analysis has the advantage that energy is fully expanded in the entire slowness domain. The slowness vector $\mathbf{p}$ for both $P$ - and $S$ waves can be obtained independently, giving us more information to investigate complicated near-source processes. The calculation based on 1D slowness analysis gives the energy as a function of horizontal slowness $p_{x}$. It provides the necessary information to separate the trapped and leaking energy. Although it does not directly give the full slowness domain information, by combining dynamic and kinematic characteristics, we can resolve the near-source phenomena in most cases without ambiguity.

Equations (8) and (9) are expressed with energy, because energy can be directly summed. However, regional phase observations are usually taken from amplitudes. To compare the numerical prediction with observations, we calculate the normalized square root of the energy

$$
A=\left(E / E_{0}\right)^{1 / 2},
$$

where $E_{0}$ is a normalization factor that can be obtained by calculating the response of a unit source. The unit source is located at unit distance in an infinitely homogeneous model. It has a unit intensity, the same source time function as that used in the simulation and its response passes through the same frequency filter. Normalized square root energy $A$ is consistent with the conventional $L g$-wave measurement based on the rms amplitude of the waveforms. Another advantage of using $A$ is that the variation of amplitude distribution is smoother than the variation of energy. For these reasons, throughout this article, we will use the normalized square root energy in all figures although we sometimes simply call it "energy."

Figure 1 shows examples of 1D and 2D slowness analyses. The energy can either be expressed as a function in 2D slowness domain, or as a function in the mixed slownessdepth domain. For references, the upper-mantle $S$ slowness $p_{\mathrm{SM}}=1 / v_{S \text {-mantle }}$, crustal $P$ slowness $p_{P}=1 / v_{P}$ and $S$ slowness $p_{S}=1 / v_{S}$ are labeled in the figure, with $v_{S \text {-mantle }}=4.57$ $\mathrm{km} / \mathrm{sec}, v_{P}=6.0 \mathrm{~km} / \mathrm{sec}$, and $v_{S}=3.5 \mathrm{~km} / \mathrm{sec}$. All $S$-wave energy with horizontal slowness larger than the uppermantle $S$ slowness, whether directly radiated from the source or generated as secondary phases, will be trapped in the crustal wave guide and will contribute to the guided regional 
(a)

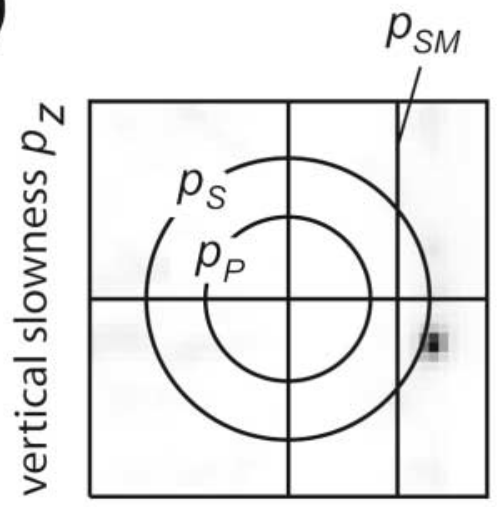

horizontal slowness $p_{X}$ (b)

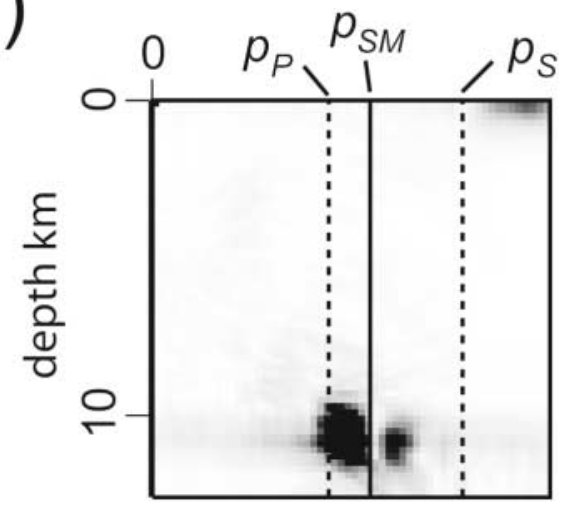

horizontal slowness $p_{x}$

Figure 1. Slowness domain display with (a) energy distribution in 2D slowness domain and (b) energy distribution in mixed horizontal slowness and depth domain. Vertical lines indicate the upper-mantle $S$ slowness $p_{\mathrm{SM}}$. Circles and dashed lines denote crustal $P$-wave slowness, $p_{P}$, and $S$-wave slowness, $p_{S}$. Energy falling to the right of upper-mantle $S$ slowness can be trapped in the wave guide and contribute to $L g$.

phases unless subsequent scattering causes it to leak out. Figure 2 is a sketch showing the configuration of the FDSA method for investigating the near-source processes. The model uses an explosion source, a fine-scale near-source velocity model, and a short-distance receiver array to provide synthetic seismograms for the ensuing slowness analysis.

Figure 3 gives an example of slowness analysis. Shown on the top is the synthetic seismogram at the center of the mini array. In the middle are energy distributions for $P$ coda, $L g$ and $R g$ waves in 2D slowness domain. These energy distributions clearly show that the $P$ coda is composed of $P$ and reflected $p S$ waves. Both of these have relatively small horizontal slowness. The $L g$ wave is composed of multiply reflected $S$ waves. Its energy falls on the $S$-wave slowness circle, and part of the energy stays on the right of the uppermantle $S$ slowness and forms trapped phases. The $R g$ wave is also a trapped mode with horizontal slowness larger than the $S$ slowness. Shown at the bottom of Figure 3 is the energy isolated by the slowness analysis. The horizontal axis is time or, equivalently, the inverse of the group velocity. The vertical coordinate is the horizontal slowness or, equivalently, the apparent horizontal-phase velocity. The filled circles are energy measured in the slowness domain with their sizes being proportional to the amount of energy. The horizontal dashed line marks the upper mantle $S$ slowness that divides the trapped and leaky energy.

\section{Testing the Validity of the Method}

We will first check the validity of the FDSA method by comparing the energy partitioning predicted at short distances using slowness analysis with the energy measured from long-distance surface receivers via a conventional method. For all numerical examples calculated in this article,

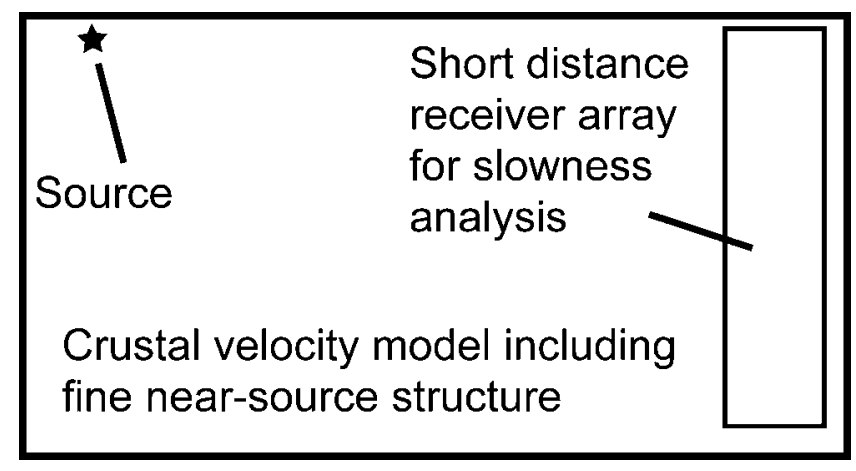

Figure 2. Configuration for using the FDSA method to investigate near-source processes.

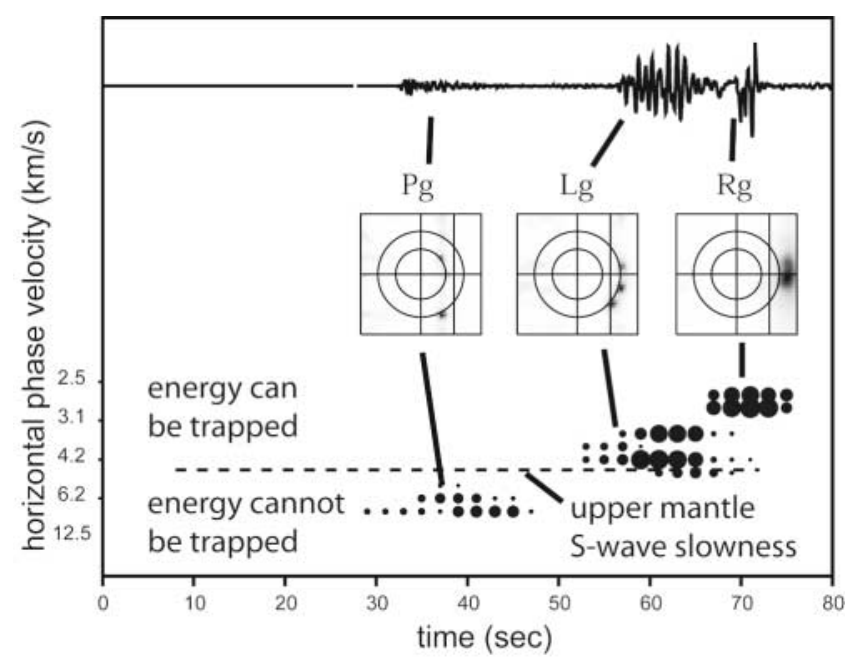

Figure 3. Example of slowness analysis at $180 \mathrm{~km}$ distance. 
unless otherwise indicated, we use the horizontally layered Eastern Kazakh (EK) model (Priestley et al., 1988) as the background and modify it by adding random velocity fluctuations at different locations. The EK model (Table 1) has a high-velocity top layer with $v_{P}=5.05 \mathrm{~km} / \mathrm{sec}$ and an upper-mantle $S$-wave velocity $v_{S \text {-mantle }}=4.57 \mathrm{~km} / \mathrm{sec}$. For this layered model, $P$ waves radiated from an explosive source cannot be effectively converted to trapped $S$ waves. The random velocity perturbations added have an exponential power spectrum with horizontal and vertical correlation lengths equal to $0.5 \mathrm{~km}$. We call the part of the model with velocity perturbations the "random patch." Within the patch, both $P$ - and $\mathrm{S}$-wave velocities have the same relative rms perturbation, and the relative density perturbation is $50 \%$ of the relative velocity perturbation. To eliminate the possible effect of sharp edges of these random patches, a space window with smoothed edges is applied to the patch. The sizes, locations, and the rms perturbations of these patches will vary depending on the purpose of the investigation.

We first test models with scattering at different depths by varying the location of random patches within the EK model. These patches are $2.5 \mathrm{~km}$ in vertical extent, extend horizontally from 5 to $25 \mathrm{~km}$, and are located at different depths. Within the patches, the $P$ - and $S$-wave velocities have $10 \%$ rms fluctuations. An explosion source is located at depth $0.5 \mathrm{~km}$. Figure 4 shows the slowness analysis results at a distance of $180 \mathrm{~km}$ in the crustal wave guide. Panels a and $\mathrm{b}$ are for frequencies $0.3-1.5 \mathrm{~Hz}$ and $2.0-5.0 \mathrm{~Hz}$, respectively. Each panel is similar to the example shown in Figure 3. The top row is for the background velocity model and the other rows are calculated for models with nearsource random velocity perturbations. The depths of the random patches are labeled in the figure. We first focus on the low-frequency results in Figure 4a. For the background velocity model, there is a strong $R g$ phase but very little energy within the $L g$ group velocity window of $3.0-3.5 \mathrm{~km} / \mathrm{sec}$, which is typical for a crustal model with a high-velocity top layer. For models with random velocity patches, compared with the background model, considerable energy is transferred to the $L g$-wave through scattering, while the $R g$ wave is weakened. The shallower the random patch, the more en-

Table 1

Eastern Kazakh Model

\begin{tabular}{cccc}
\hline $\begin{array}{c}\text { Top of Layer } \\
(\mathrm{km})\end{array}$ & $\begin{array}{c}V_{P} \\
(\mathrm{~km} / \mathrm{sec})\end{array}$ & $\begin{array}{c}V_{S} \\
(\mathrm{~km} / \mathrm{sec})\end{array}$ & $\begin{array}{c}\rho \\
\left(\mathrm{g} / \mathrm{cm}^{3}\right)\end{array}$ \\
\hline 0.0 & 5.05 & 2.91 & 2.70 \\
1.0 & 5.66 & 3.21 & 2.70 \\
5.0 & 5.55 & 3.17 & 2.70 \\
10.0 & 6.01 & 3.41 & 2.70 \\
15.0 & 6.29 & 3.55 & 2.90 \\
25.0 & 6.65 & 3.73 & 2.90 \\
30.0 & 6.89 & 3.85 & 2.90 \\
45.0 & 7.59 & 4.20 & 3.00 \\
47.5 & 8.33 & 4.57 & 3.30 \\
\hline
\end{tabular}

ergy is scattered into $L g$. For the EK model, which has a thick, high-speed crust, the distance for $P n$ to cross $P g$ at the free surface is about $200 \mathrm{~km}$. At $180 \mathrm{~km}$ distance, there is no prominent $P n$ energy shown in this analysis.

For high-frequency results in Figure $4 \mathrm{~b}$, from the top panel we see strong $P g$ energy. There is also energy within the $L g$-group velocity window. However, this energy has a similar horizontal slowness to the $P g$ wave, implying that it is generated from $P g$ through $P$-to- $P$ and $P$-to- $S$ reflections on the free surface and interfaces such as the Moho discontinuity. Although the energy exists at short distances, the steep incident angle causes energy to gradually leak to the upper mantle through multiple reflections and it cannot form trapped regional phases. In the other panels, after adding random velocity perturbations in the near-source region, part of the $P$ energy transfers to the $L g$ wave, i.e., energy falls into the proper group velocity and slowness windows. In general, the scattering affects $R g$-to- $L g$ energy at low frequencies and $P$-to- $L g$ coupling at high frequencies. Summing up energy through the wave-guide cross section and within the proper time (group velocity) windows allows the energy for the related wave types to be obtained. Specifically, summing up the energy located within the $L g$-group velocity window and above the upper-mantle $S$ slowness allows $L g$ energy at long distances to be predicted. It is necessary to confirm this calculation.

Figure 5 compares the wave-guide energy obtained using different methods. The left panel shows the square root energy passing through the wave-guide cross section at $180 \mathrm{~km}$ calculated from the slowness analysis shown in Figure 4. The right panel shows the rms amplitudes at $450 \mathrm{~km}$ calculated by using a conventional processing technique (i.e., computing surface synthetic seismograms, applying frequency filters and group velocity windows to isolate different phases, and calculating rms amplitudes for these phases). The same velocity model used in Figure 4 is adopted here and a full-scale FD simulation is computed to provide data up to $500 \mathrm{~km}$ distance. For simplicity, we label the predicted trapped energy as "Lg" energy. The relative energy changes of $P g, L g$, and $R g$ waves, as functions of the depth of the random velocity patches are shown in Figure 5. The calculations for different phases demonstrate that the relative change of energy obtained in the wave-guide slowness analysis corresponds closely to that obtained on the free surface, even for dramatic changes such as the strong scattering of $R g$. The results confirm that the slowness analysis within the wave guide correctly predicts the surface regional observations at greater distances.

To examine the energy-flux measurements obtained at even shorter distances, we compare the slowness analysis measurements at $50 \mathrm{~km}$ with those at $100 \mathrm{~km}$. For these two distances, Figure 6 gives examples of horizontal slowness analyses, which show quite different features in the slowness-depth domain because of evolution of the wave field with range. Figure 7 compares the corresponding waveguide energy measured at these two distances for different 
(a) $0.3-1.5 \mathrm{~Hz}$ group velocity $\mathrm{km} / \mathrm{s}$
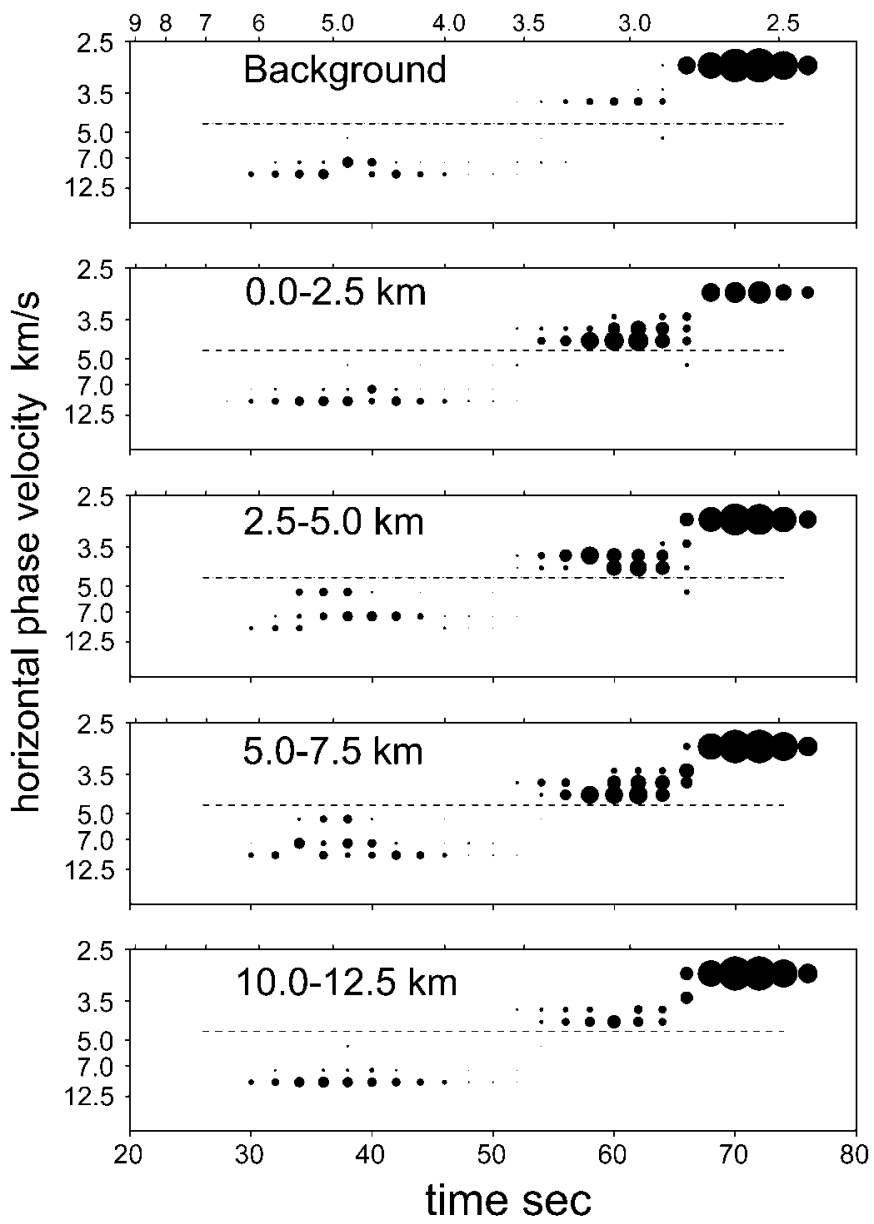

(b) $2.0-5.0 \mathrm{~Hz}$ group velocity $\mathrm{km} / \mathrm{s}$
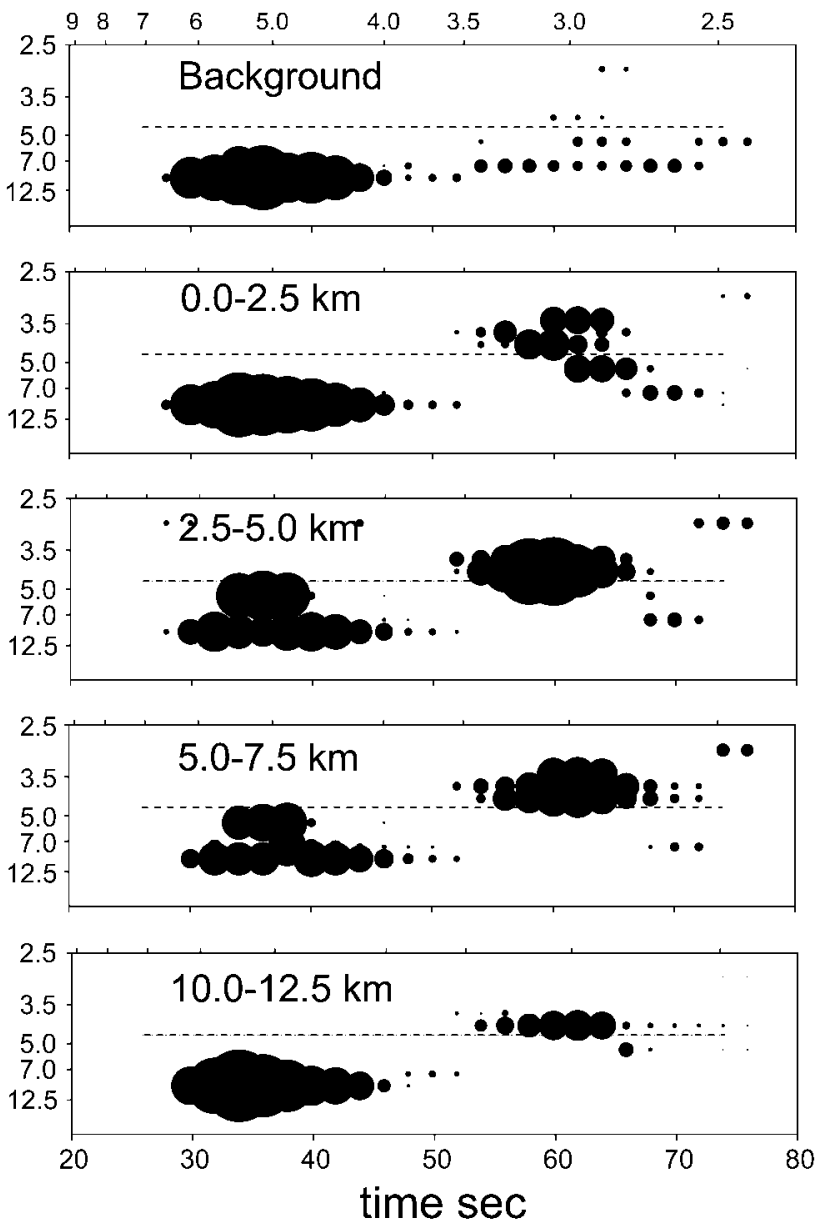

Figure 4. Slowness analysis calculated for the EK model and EK model with random patches at different depths: (a) Frequency band 0.3-1.5 Hz. (b) Frequency band 2.0$5.0 \mathrm{~Hz}$. The top row is for a background velocity model and the lower rows are for models with random patches. The depths of random patches are labeled in the panels.

For details, see the text.

frequency bands. The vertical and horizontal coordinates are for measurements at the two distances, and dots represent results for different source depths and velocity models. Although a wide range of near-source structures and source depths are used to generate these measurements, the results show a general linear relationship for all frequency bands. This further verifies that we can use a small model to investigate the near-source energy partitioning robustly.

\section{Investigating Regional Phase Excitation}

\section{$P$-pS-to- $L g$ and $P$-to- $L g$ Conversion}

We first investigate the $P$ - $p S$-to- $L g$ conversion caused by near-source lateral velocity variations and assess its effect on the explosion $S$-wave energy budget. In a horizontally layered model with overburden $P$-wave velocity larger than the upper-mantle $S$-wave velocity, the free-surface-reflected $p S$ wave has a steep incidence angle and cannot be trapped in the crustal wave guide to form $L g$. In this case, the energy transfer through $P$ - $p S$-to- $L g$ coupling is almost zero. Although, in general, it is agreed that the existence of nearsource lateral velocity variation can increase the $P$-to- $L g$ energy exchange, the detailed mechanism underlying this process is still not fully understood.

Figure 8 compares the simulated $P$-pS-to- $L g$ coupling in models with and without near-surface lateral velocity variations. Figure 8a is for the EK model. A shallow explosion source located at depth $0.5 \mathrm{~km}$ generates $P, p S$, and $R g$ waves. Two-dimensional slowness analysis is conducted for selected phases in the wave field and the results are shown together with the wave-field snapshot. The synthetic seismograms were bandpass filtered between 2.0 and $6.0 \mathrm{~Hz}$ before the slowness analysis. As seen from the result, the $P$ 


\section{waveguide energy flux at $180 \mathrm{~km}$}

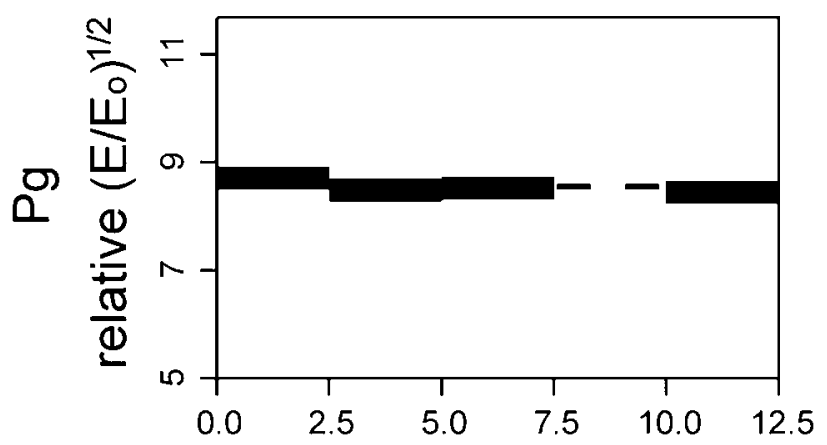

\section{surface energy at $450 \mathrm{~km}$}

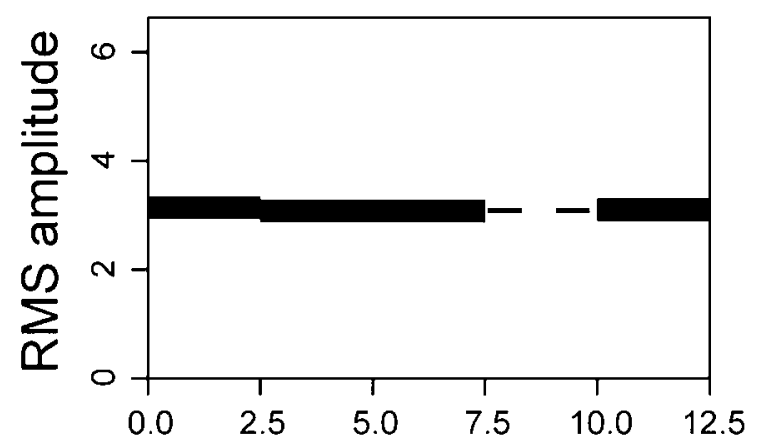

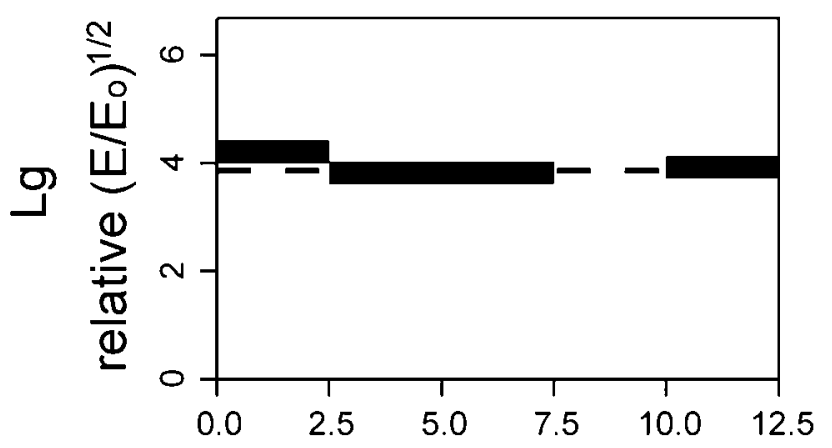
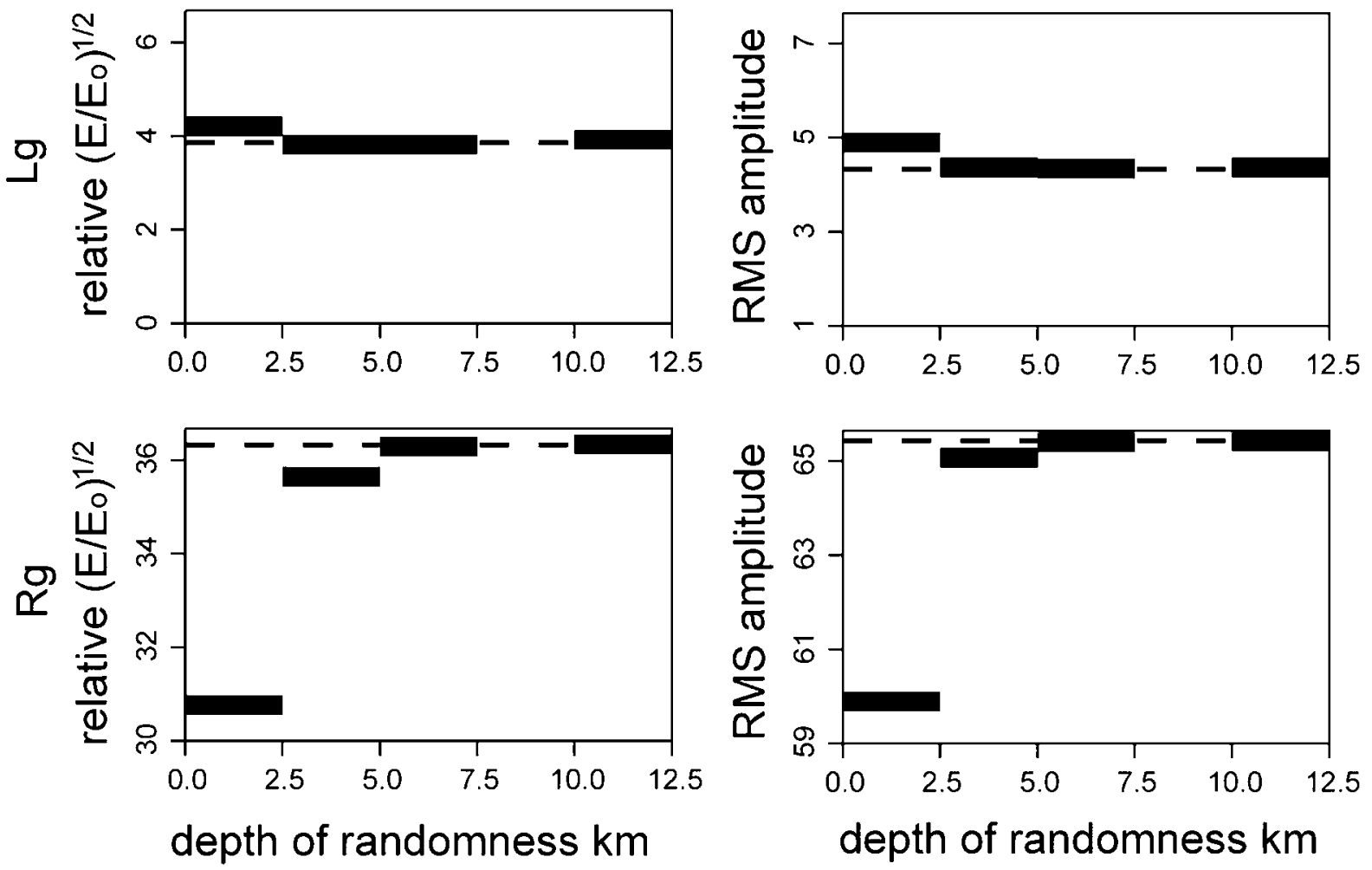

Figure 5. Comparison between wave-guide energy flux at $180 \mathrm{~km}$ (left column) and the wave energy on the surface at $450 \mathrm{~km}$ (right column) for $P g, L g$ and $R g$ windows. The frequency range is $0.3-1.2 \mathrm{~Hz}$. Shown in each panel is relative energy versus the depth of random patches. Dashed lines indicate the energy level for the background model and short bars indicate the energy changes due to the near-source scattering.

wave leads the wave field and has a distinct slowness. Reverberations within the uppermost crust causes multiply parallel $p S$ wavefronts with their horizontal slowness approximately equal to the overburden $P$ slowness. The $p S$ energy stays to the left of the upper-mantle $S$ slowness and there is no energy transferred from $P$ to $L g$. In Figure 8b, a shallow random velocity patch is added to the EK model to test the effect of near-surface scattering. The random patch has a 5\% $\mathrm{rms}$ velocity fluctuation and is located at distances $5-15 \mathrm{~km}$ and depths $0-2.5 \mathrm{~km}$ (shown in the snapshot as a shaded area). The slowness analyses are conducted for $P, p S$, and $p S$ coda. Although $P$ and early $p S$ waves are barely affected, the $p S$ coda clearly contains some scattered energy with horizontal slowness to the right of the upper-mantle $S$ slowness.

To investigate further the scattering from a shallow random patch, horizontal slowness analysis is conducted at a distance of $20 \mathrm{~km}$ and a depth of $0-12.5 \mathrm{~km}$. Figure 9 shows the energy distribution in the slowness-depth domain with 


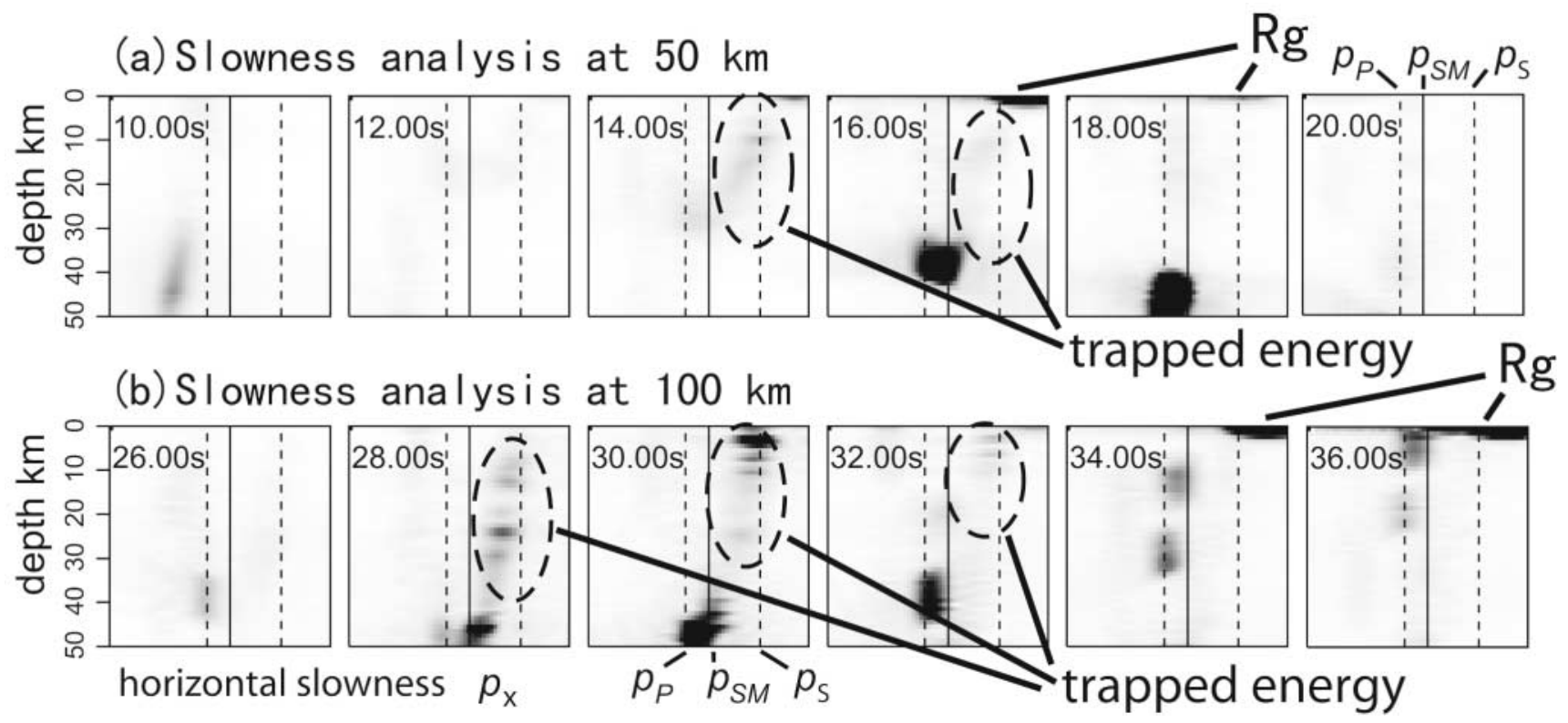

Figure 6. Examples showing the slowness analyses at distances $50 \mathrm{~km}$ (a) and 100 km (b). The $p_{S M}, p_{P}$, and $p_{S}$ are upper-mantle $S$ slowness, crustal $P$ slowness, and $S$ slowness, respectively. The energy that can be trapped in the crustal wave guide is indicated in the figure.
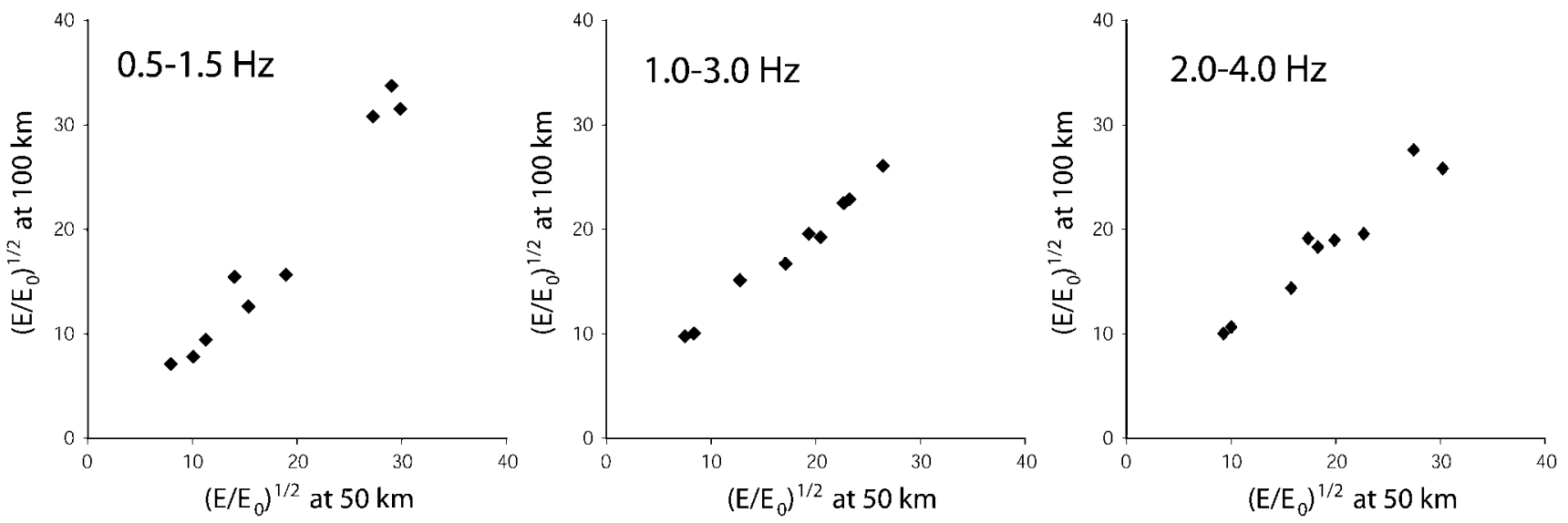

Figure 7. Comparison between trapped wave-guide energy measured at $50 \mathrm{~km}$ (horizontal coordinate) and $100 \mathrm{~km}$ (vertical coordinate) for frequency bands $0.5-1.5 \mathrm{~Hz}$, 1.0-3.0 Hz, and 2.0-4.0 Hz. Different dots are results from different velocity models and source depths. The results show a general linear relationship for all frequencies.

arrival times and major phases labeled in the frames. The two prominent downgoing phases are the $P$ wave and the free-surface-reflected $p S$ wave. The $R g$ energy enters the array at $6.0 \mathrm{sec}$ with its depth close to the surface and slowness beyond the $S$ slowness. Figure 9a shows the result using the EK model. Due to the nearly horizontal propagation of the $P$ wave at the free surface, the $p S$-wave energy has a horizontal slowness that is similar to the overburden $P$ slowness and the energy falls to the left of the upper-mantle $S$ slowness. In Figure $9 b$ and c, shallow random velocity patches with rms velocity fluctuations $3 \%$ and $5 \%$ are added to the EK model at distances 5-15 km and depths $-2.5 \mathrm{~km}$ (the same position as in Fig. 8b). As seen in the figure, scattering causes part of the $p S$ energy to cross the upper mantle $S$ slowness and build up in the dashed rectangles. At shallow depths, the slowness of the scattered energy approaches the $S$ slowness. With an increase in depth, the slowness of this energy gradually merges with the $P$ slowness. The slowness behavior is consistent with scattering of waves at shallow depths, increasing as shallow heterogeneity increases.

Figure 10 investigates scattering taking place at deeper depths. The configuration of the source and model is similar to that used in Figure 8b, except the random patch with 3\% rms velocity fluctuation is added to the EK model between 
(a) EK-model

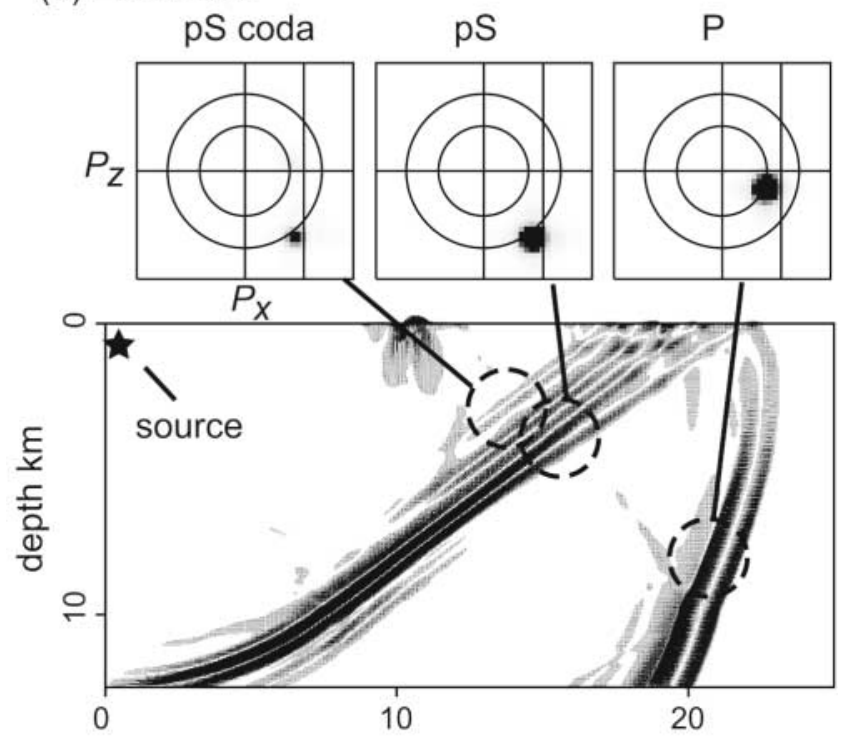

(b) EK+random

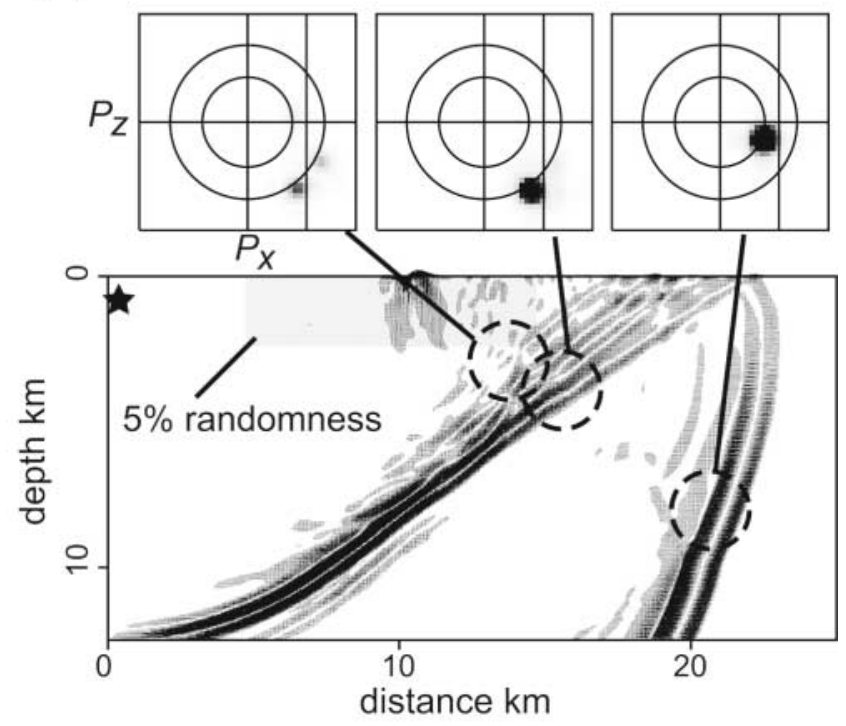

Figure 8. $\quad P$ - $p S$-to- $L g$ conversion due to shallow scattering. (a) EK model. (b) EK model with a shallow random patch. The random patch has a $5 \% \mathrm{rms}$ velocity fluctuation and is shown as a shaded area between horizontal distances of 5 and $15 \mathrm{~km}$ and between depths of 0 and $2.5 \mathrm{~km}$. The snapshots and results of slowness analyses of $P, p S$, and $p S$ coda are shown in the figure. Details are given in the text.

distances 5 and $15 \mathrm{~km}$ and depths 2.5 and $10 \mathrm{~km}$ (shown in the snapshot as a shaded area). The 2D slowness analysis is conducted for selected phases in the wave field and the results are presented in the figure. After passing through the random region, a $P$ coda is composed of scattered $P$ and $S$ waves generated from the direct $P$ wave. Although the early part of the $p S$ wave does not contribute to the trapped energy, its later part contains energy located to the right of the uppermantle $S$ slowness, which therefore will contribute to the trapped regional phases. Figure 11 gives the energy distribution in the slowness-depth domain for different models where panel $\mathrm{a}$ is for the EK model and panels $\mathrm{b}$ and $\mathrm{c}$ are for the EK model with $3 \%$ and $5 \%$ rms fluctuations in a random patch like that used in Figure 10. The slowness analysis is conducted at a distance of $20 \mathrm{~km}$ and for depths between 0 and $12.5 \mathrm{~km}$. As expected, with the EK model no energy is seen beyond the upper mantle $S$ slowness, but after the lateral velocity variations are introduced, energy starts to build up to the right of the upper-mantle $S$ slowness. Two types of scattered energy can be found in the slowness-depth domain: weak but widely distributed $S$ energy (indicated by the dashed ellipses) and scattered energy linked to the $p S$ wave (indicated by the dashed rectangles). Both types of energy satisfy the criterion $p_{x} \geq p_{S \text {-mantle }}$ and will contribute to the $L_{g}$ wave. The widely spread, scattered $S$ wave is generated by the $P$-to- $L g$ coupling through volumetric scattering. The scattering process redistributes the angle spectrum of the original incident waves. Because no lateral heterogeneity exists at the top of the crust, scattered $p S$ waves are generated by the interaction between distorted incident $P$ waves and a smooth free surface.

Comparing Figure 11 with Figure 9, the scattered $p S$ wave generated from a deeper random patch appears later than that from a shallow random patch. Both volumetric scattering and scattering near the free-surface affect the general $P$-to- $L g$ conversion.

\section{Contributions from the $\mathrm{S}^{*}$-Wave}

For shallow explosion sources, the $S^{*}$ wave may become a significant contributor to $L g$ (Gutowski et al., 1984; Lilwall, 1988; Xie and Lay, 1994; Vogfjord, 1997). The amplitude of $S^{*}$ can be large if the source depth is within a fraction of a wavelength from an interface. This makes its excitation highly dependent on the source depth and frequency. Figure 12 shows snapshots for explosion sources at $0.5 \mathrm{~km}$ and $3.0 \mathrm{~km}$, respectively. The result clearly shows that a shallow source generates larger $S^{*}$ and $R g$ waves. We investigate the contribution of the $S^{*}$ wave within the EK model. Figure 13 shows horizontal slowness analyses at a distance $35 \mathrm{~km}$ and for depths between 0 and $30 \mathrm{~km}$. The time window is chosen between 11 to $13 \mathrm{sec}$ after the direct $P$ wave passes the receiver array. The synthetic seismograms are bandpass filtered between 1.0 and $5.0 \mathrm{~Hz}$. The four rows from top to the bottom correspond to source depths $0.25 \mathrm{~km}$, $0.5 \mathrm{~km}, 1.0 \mathrm{~km}$, and $2.0 \mathrm{~km}$, respectively. The major arrival is the downgoing free-surface-reflected $p S$ wave, which has a horizontal slowness similar to the overburden $P$ slowness. As expected for a horizontally layered model, the $p S$ energy stays to the left of the upper-mantle $S$ slowness and has no contribution to the trapped regional phases. For shallow sources, the $R g$ wave enters the array at about $12 \mathrm{sec}$ and its energy concentrates between 0 and $3 \mathrm{~km}$, as can be seen on the upper-right corners in the slowness-depth domain. For source depth of $2.0 \mathrm{~km}$, the $R g$ wave is very weak. The $S^{*}$ 


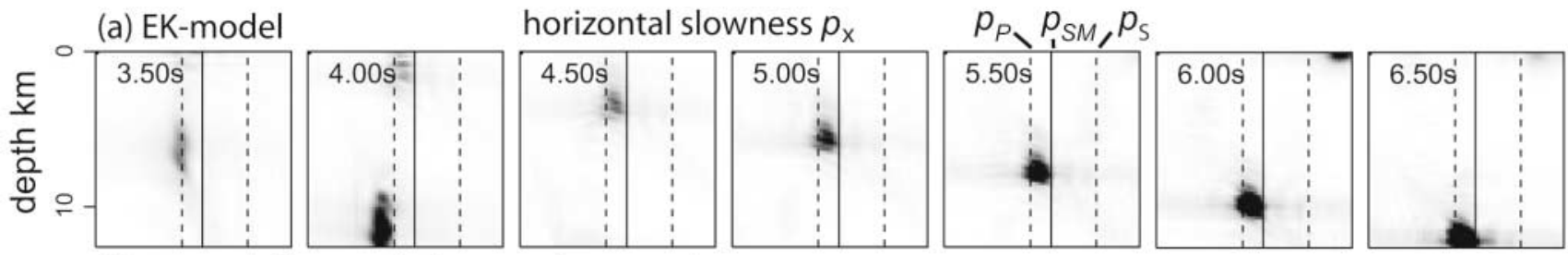

(b) EK-model $+3 \%$ shallow random patch
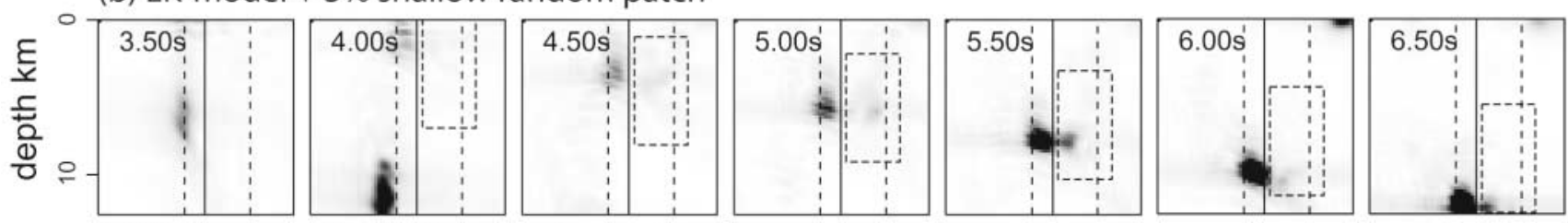

(c) EK-model $+5 \%$ shallow random patch
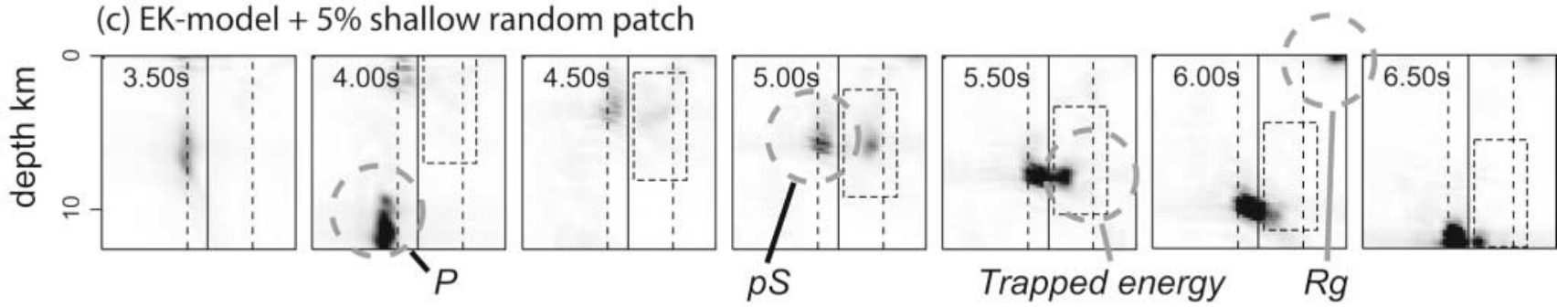

Figure 9. Horizontal slowness analyses for investigating the $P$ - $p S$-to- $L g$ coupling with (a) the EK model, (b) the EK model plus a 3\% shallow random patch, and (c) the EK model plus a $5 \%$ random patch. The $p_{S M}, p_{P}$, and $p_{S}$ are upper-mantle $S$ slowness, crustal $P$ and $S$ slowness, respectively. The slowness analyses are conducted at a distance of $20 \mathrm{~km}$ and a depth range of $0-12.5 \mathrm{~km}$. The configuration of the source and model is the same as that used in Figure 8. Major phases are labeled in the figure and energy circled by dashed rectangles is the scattered $p S$ wave.

wave enters the array from a shallow depth and gradually merges with the $p S$ wave (also refer to Fig. 12). The $S^{*}$ wave is strong for shallow sources and its amplitude decreases with increasing source depth. Very little $S^{*}$ energy can be observed for source depths below $2 \mathrm{~km}$. In the joint domains, the $S^{*}$ energy can be isolated and quantified even within a complicated wave field, which is very difficult with remote surface synthetics. The dashed rectangles are the time-slowness-depth window used to locate the $S^{*}$ energy. The time window is chosen after the arrival of direct $P$ wave, and a variable depth range is chosen to avoid contamination from the $R g$ wave. The slowness range is chosen between 0.23 and $0.34 \mathrm{sec} / \mathrm{km}$. The energy from successive windows can be summed together to give the contribution of $S^{*}$ to the trapped regional phases.

\section{The Frequency-Dependent $L g$ Excitation Function}

The frequency dependence of $L g$-wave excitation is rooted in the underlying physical processes and is usually controlled by different characteristic scales. For example, the excitation of $L g$ by $S^{*}, R g$-to- $S$ scattering and spall are all highly source depth dependent. The excitation spectra from individual or joint mechanisms contributing to regional phases depict the frequency dependence of these processes. Frequency-dependent $P / S$ ratios will depend on the excita- tion functions of multiple phases. We use FDSA to quantify $L g$-wave excitation spectra from $S^{*}$ and $p S$ waves. Figure 14 gives the $S^{*}$-to- $L g$ excitation spectra as functions of source depth and frequency. The slowness analysis and multidomain window used to pick the trapped energy are similar to that shown in Figure 13. A series of bandpass filters is used to give responses at different frequencies. The vertical coordinate is the normalized relative energy $\left(E / E_{0}\right)^{1 / 2}$. Because the source time function has been taken away, the excitation function is the impulse response of the model to the source. The results clearly show that the $S^{*}$-to$L g$ excitation is generally enhanced for lower frequency and shallow source depth. The major contribution comes from sources located above $1 \mathrm{~km}$. For sources at depths below $1 \mathrm{~km}$, only low-frequency energy below $1 \mathrm{~Hz}$ has significant contribution to $L g$-wave excitation. However, the responses are also model dependent. For a model with a homogeneous crust (Fig. 14a), the distribution has simple monotonic tendencies in both source depth and frequency. For the EK model (Fig. 14b), the excitation spectrum has a maximum at depth $1 \mathrm{~km}$ and a more complicated frequency dependence. This may reflect the fact that the EK model has an interface at $1 \mathrm{~km}$ depth. The $S^{*}$ waves generated or reflected from multiple interfaces may interfere with each other and give a complicated frequency spectrum.

To investigate the combined effect for $S^{*}$ wave and 


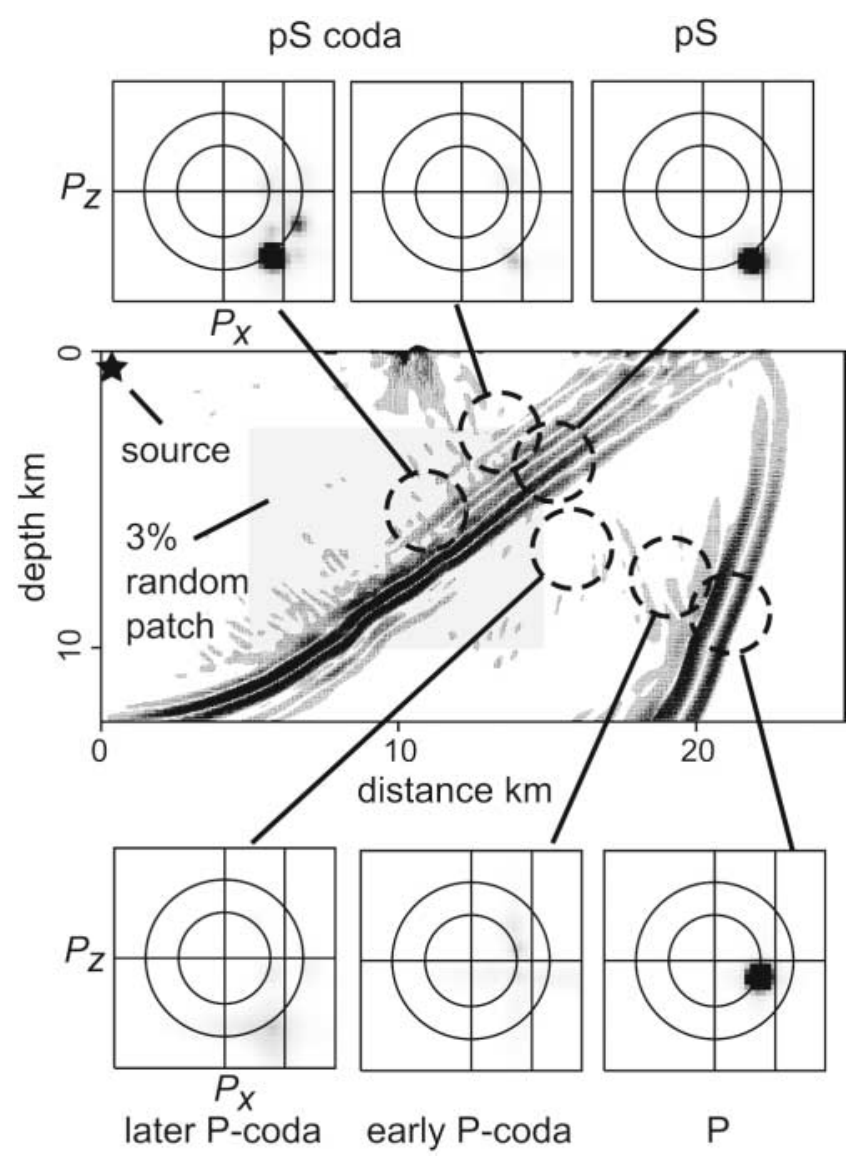

Figure 10. The $P$ - $p S$-to- $L g$ conversion due to a deeper random patch. The random patch has a $3 \% \mathrm{rms}$ velocity fluctuation and is shown as a shaded region between distances 5 and $15 \mathrm{~km}$ and depths 2.5 and $10 \mathrm{~km}$. The slowness analysis for $P, P$ coda, $p S$ and $p S$ coda are also shown in the figure.

near-source scattering, we add shallow random-velocity patches to the EK model. The random patch extends between distances of 5 and $25 \mathrm{~km}$ and depths of 0 and $2.5 \mathrm{~km}$. Shown in Figure 15a and $\mathrm{b}$ are excitation spectra for random patches with rms velocity fluctuations of $3 \%$ and $5 \%$, respectively. The most prominent feature is the buildup of high-frequency energy. The scattered energy increases with $\mathrm{rms}$ velocity fluctuations. Figure $15 \mathrm{c}$ and d isolates the scattered energy by subtracting the excitation spectrum of the EK model from the spectra for models with random velocity patches. Two types of energy can be identified within the frequency-depth domain. The high-frequency energy results from $P-p S$-to- $L g$ and $P$-to- $L g$ scattering. This energy is especially important for deeper sources to generate $L g$ waves, because a deeper source generates little trapped energy in a horizontally layered model. The low-frequency energy concentrated at shallow source depths comes from $R g$-to- $L g$ scattering.

Figure 16 gives the excitation spectra for the EK model with deeper random patches. The random patch is located between distances of 5 and $25 \mathrm{~km}$ and depths of 7.5 and 10.0 $\mathrm{km}$. Figure 16a and $\mathrm{b}$ gives excitation spectra for random patches with rms velocity fluctuations of $3 \%$ and $5 \%$, respectively. Figure 16c and d gives the isolated scattered energy. The scattered energy from the deeper random patches has little low-frequency content, which supports the interpretation that the low-frequency energy comes from the $R g$ to- $L g$ scattering. The frequency-dependent excitation spectra establish the relationship between the observations and the characteristics of sources and near-source structures. They provide the basis for evaluating the dominant mechanisms for $L g$-wave excitation.

Xie (2002) investigated the $P n$ and $L g$ spectra from a group of explosions and found that the difference between these spectra requires a nonflat transfer function between the two phases. The $L g$-wave excitation function obtained in this article is the impulse response. To compare with the observed $L g$ spectrum, the source spectrum should be added. Assuming that the Pn spectrum roughly represents the source spectrum, the excitation function obtained here approximates the $P n$-to- $L g$ transfer function. Qualitatively, our excitation function explains the observations of Xie (2002) which require a nonflat $P n$-to- $L g$ transfer function with an enhanced low-frequency excitation for $L g$. To make a quantitative comparison, additional investigation is required.

\section{Discussion and Conclusions}

A FD modeling plus slowness analysis (FDSA) method has been developed to investigate near-source energy partitioning and $L g$-wave excitation of explosive sources. The method has two major advantages. First, it allows us to study the near-source processes in multiple domains, including space, time, slowness, and frequency. This provides an opportunity to isolate different mechanisms within the complex near-source environment. Second, the FDSA method can be applied at a close range, well before the $L g$ wave is actually formed. It provides us with uncontaminated near-source information by calculating a relatively small velocity model with very fine near-source structures. Because this is a very efficient method, we can use it to investigate a broadfrequency band and to test a large number of source model parameters. As examples, we investigated the contributions of $P$ - $p S$-to- $L g$ conversion and $S^{*}$-to- $L g$ excitation using models with near-source random velocity fluctuations. The contribution of $S^{*}$-to- $L g$ is concentrated at low frequencies and occurs for very shallow source depths. The contribution of $P$ - $p S$-to- $L g$ coupling in the presence of near-source smallscale random heterogeneities is concentrated at high frequencies. The excitation spectra of these mechanisms were calculated.

There are other potential $L g$ excitation mechanisms (e.g., $R g$-to- $L g$ scattering, spall, and tectonic release), which were not systematically investigated in this study. The ability to handle a broad-frequency band makes the FDSA an ideal tool to investigate excitation spectra and $P / S$-type spectra ratios for different mechanisms and source model parameters. These spectra and spectra ratios form the basis of most 

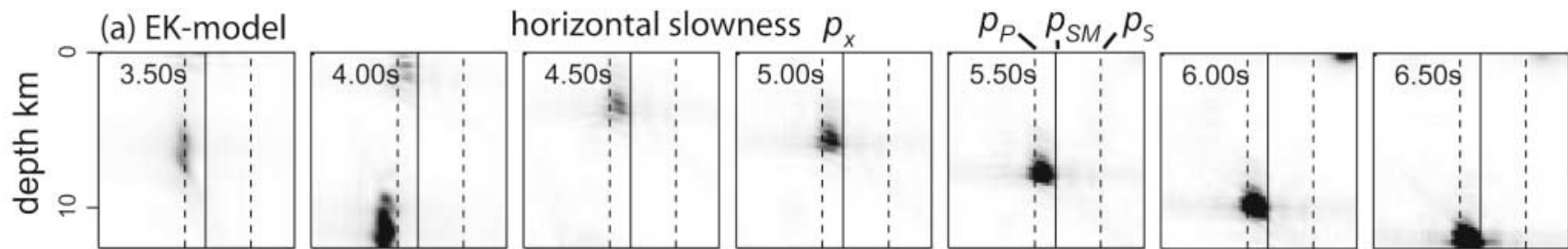

(b) EK-model + 3\% deep random patch
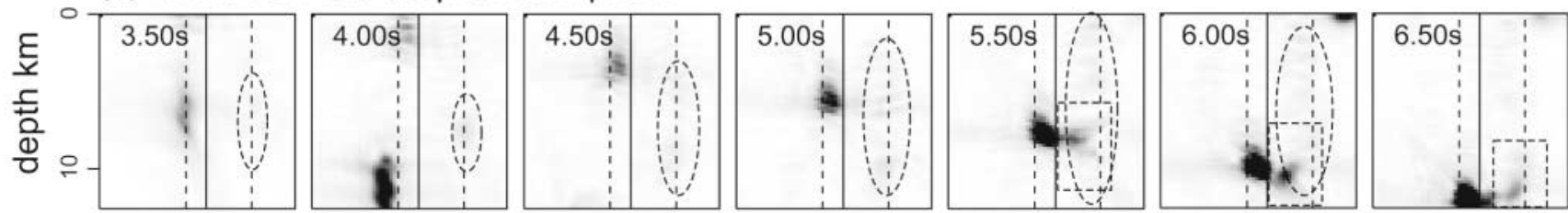

(c) EK-model $+5 \%$ deep random patch
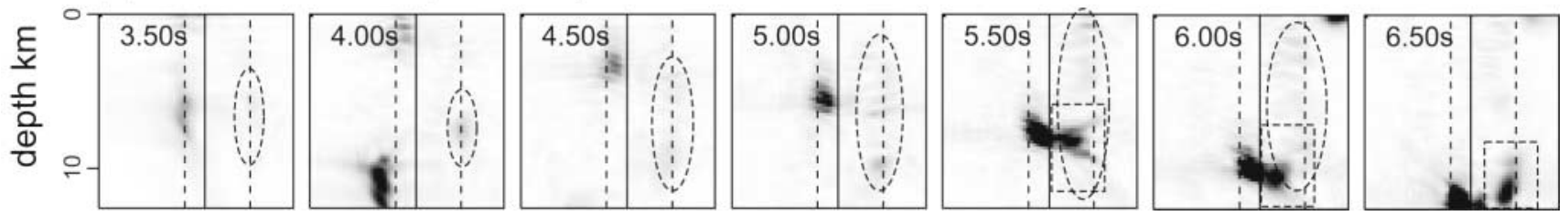

Figure 11. Energy distribution in depth and horizontal slowness domain for (a) the EK model, (b) EK model plus a 3\% random patch, and (c) EK model plus a 5\% random patch. The configuration of the source and model is same as that used in Figure 10. Energy circled by dashed rectangles is $P$-pS-to- $L g$ scattering, and energy circled by dashed ellipses is $P$-to- $L g$ scattering.

(a) source depth $0.5 \mathrm{~km}$

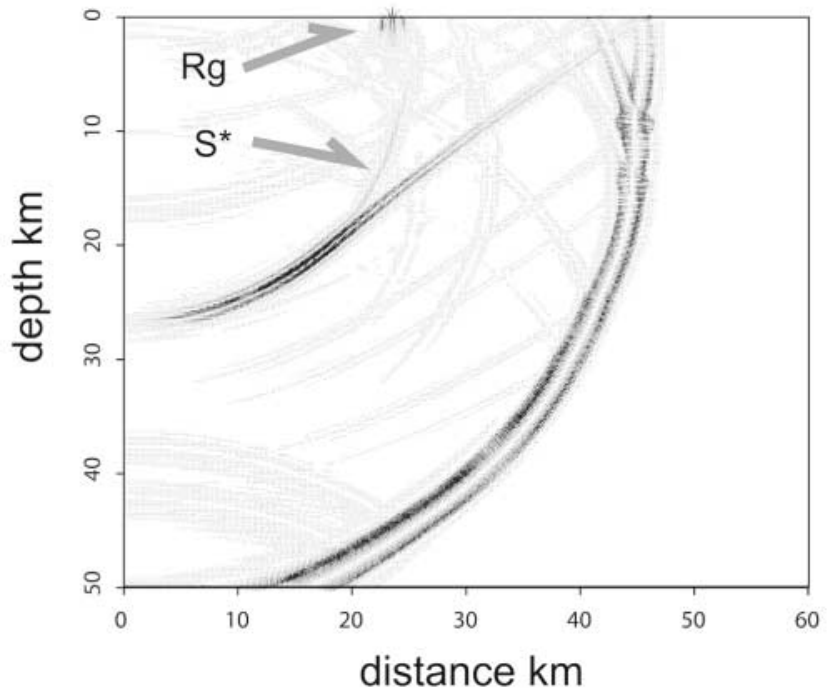

(b) source depth $3.0 \mathrm{~km}$

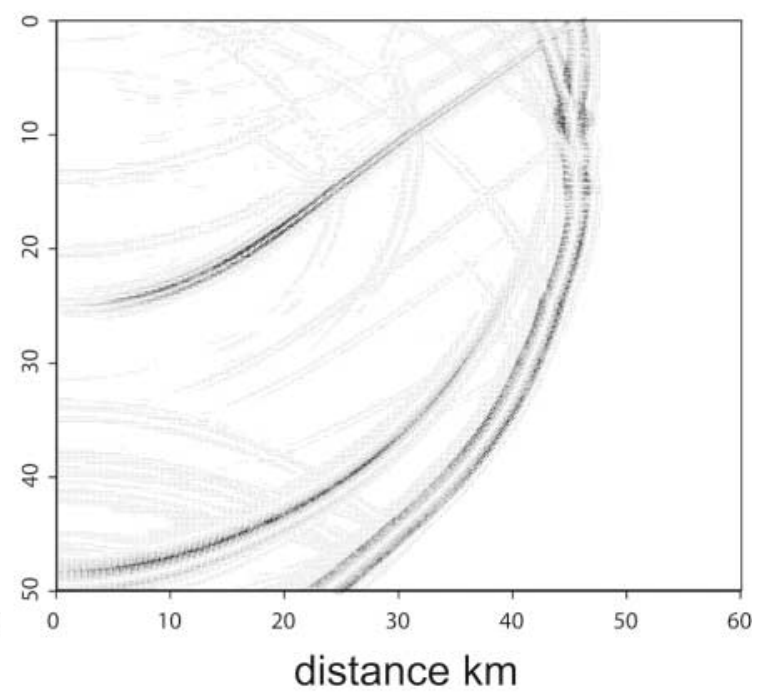

Figure 12. Wave-field snapshots for explosion sources at depths $0.5 \mathrm{~km}$ (a) and 3.0 $\mathrm{km}(\mathrm{b})$. Note that a shallower explosion is a more efficient source for generating $S^{*}$ and $R g$ waves.

regional identification discriminants. Numerical modeling can establish a link between the physical model and observable frequency-dependent features and place regional event identification on a sound physical basis. Scattering from uneven boundary topography should be included in future studies. Although most $\mathrm{Lg}$ observations are from vertical- component seismograms, the tangential component often has as much energy as the vertical component. Because 2D geometry decouples the $P-S V$ problem from the $S H$ problem, it does not provide any information on the coupling between the source and the $\mathrm{SH}$ component. The future development of a 3D FDSA method is thus motivated. 


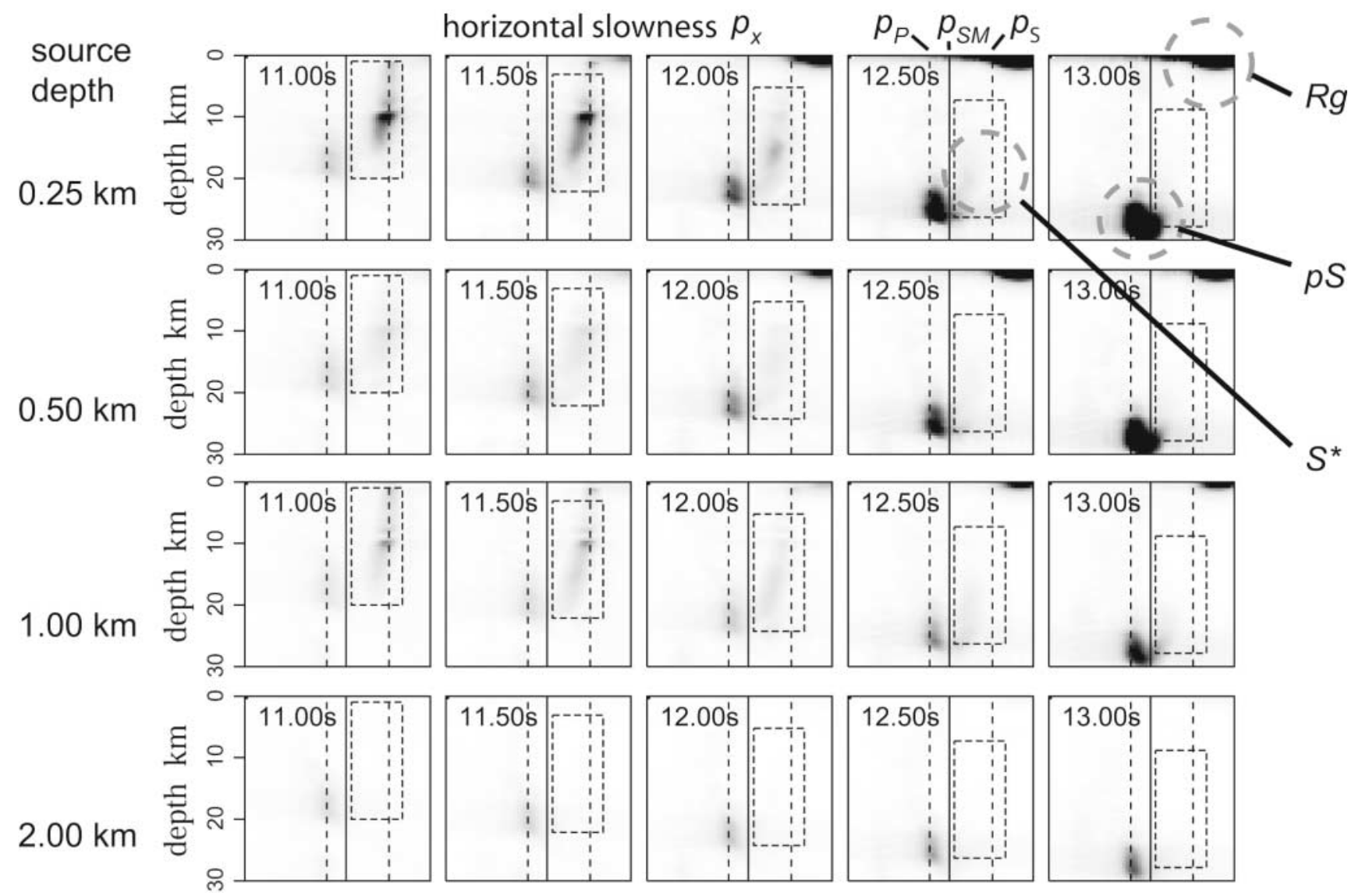

Figure 13. Slowness analysis for investigating $S^{*}$-to- $L g$ conversion. Different rows are for different source depths. Dashed rectangles indicate the time-space-slowness windows used to pick the $S^{*}$ energy.

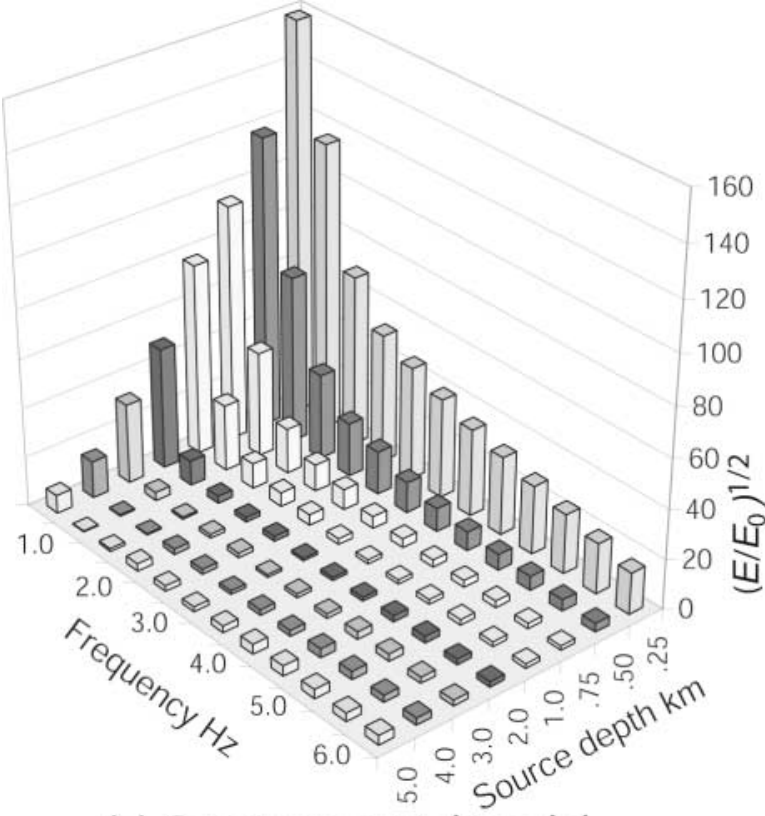

(a) Constant crustal model

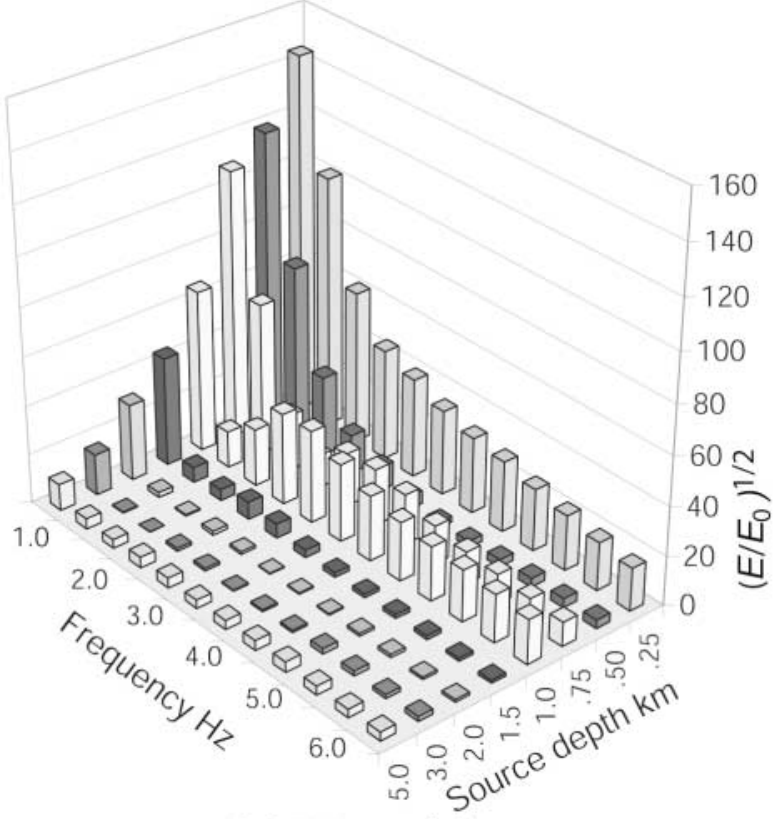

(b) EK-model

Figure 14. Normalized $L g$-excitation spectra for sources in different velocity models and at different depths with a model with a homogeneous crust (a) and the EK model (b). 


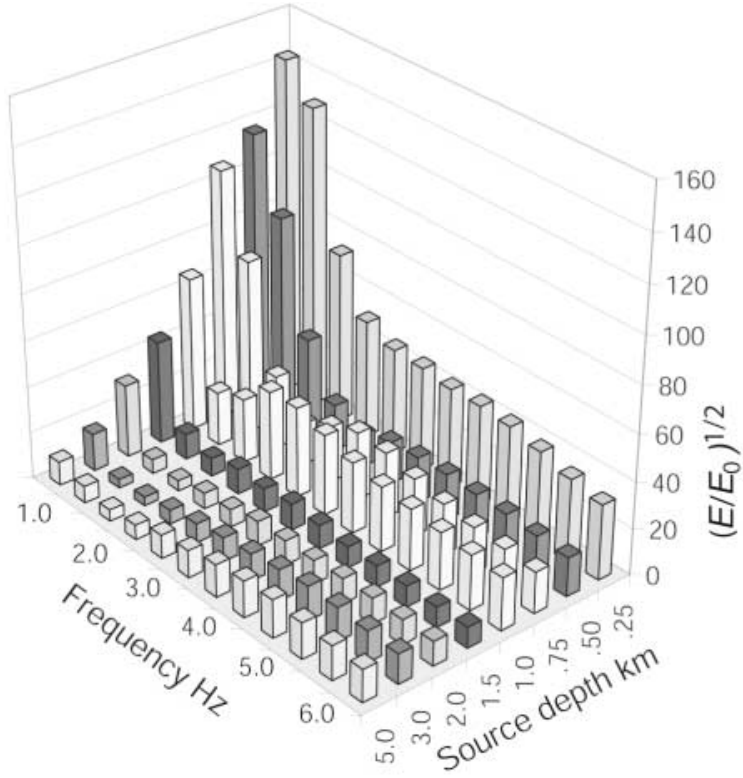

(a) EK $+3 \%$ shallow random

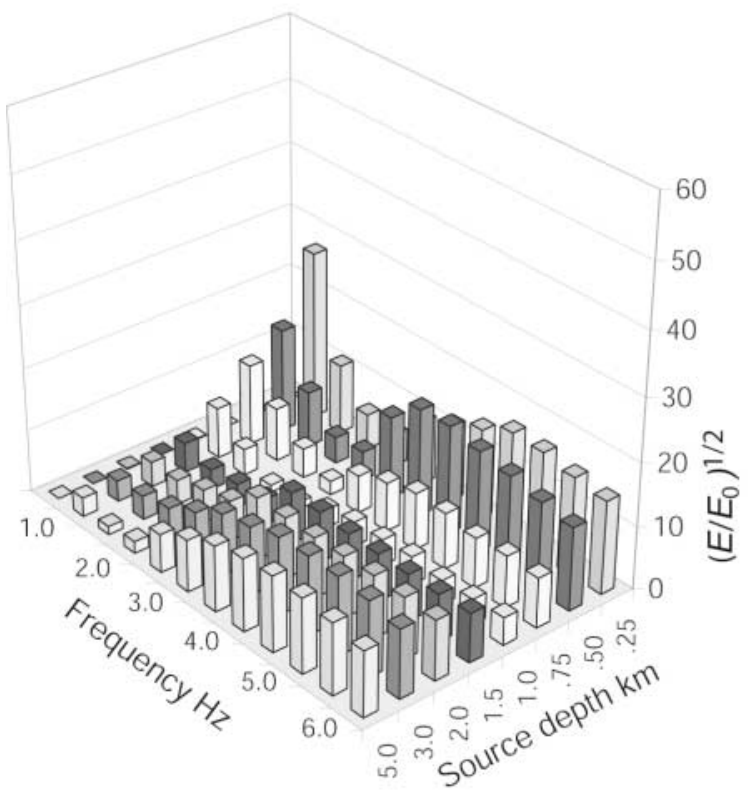

(c) EK+3\% scattered energy

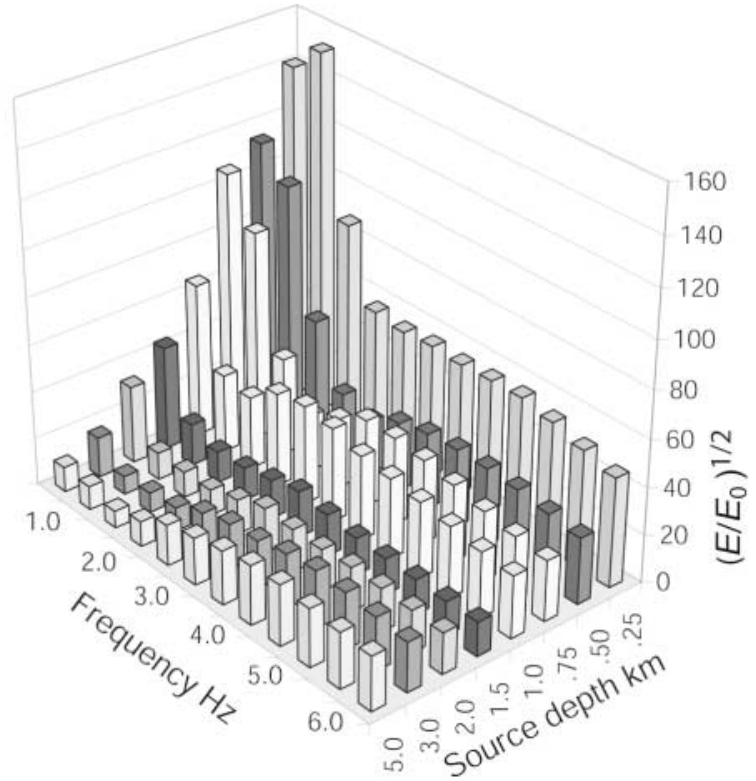

(b) EK $+5 \%$ shallow random

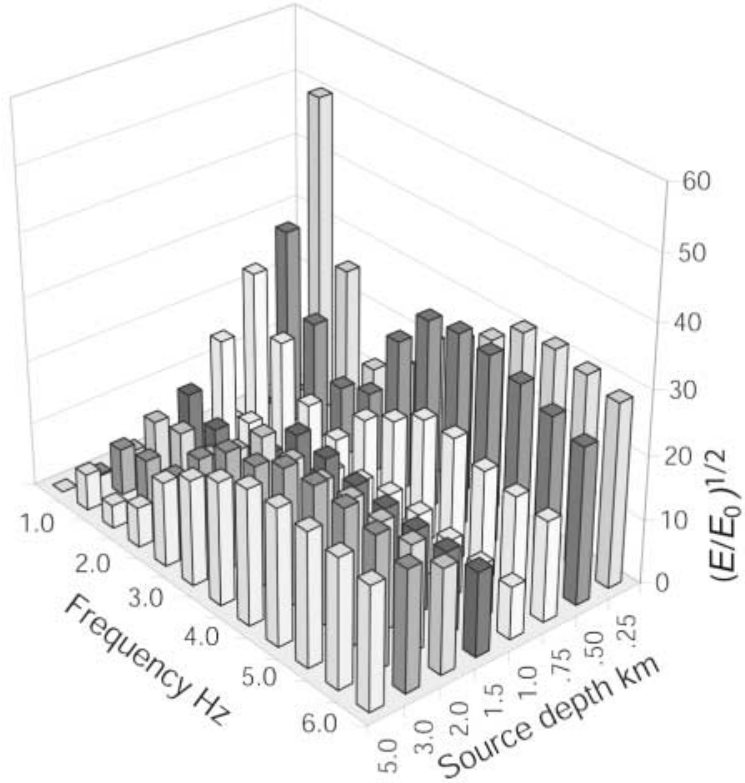

(d) EK+5\% scattered energy

Figure 15. Normalized $L g$-excitation spectra for sources in the EK model with shallow random patches, for (a) the EK model with a 3\% shallow random patch, (b) the EK model with a 5\% shallow random patch, (c) and (d) the isolated scattered energy in (a) and (b), respectively, due to the random patches found by removing the energy for the layered models. Note different vertical scales are used for scattered energy.

\section{Acknowledgments}

The authors thank Dr. Ru-Shan Wu for many discussions on $L g$-wave scattering in random media. We sincerely thank Dr. Jiakang Xie and an anonymous reviewer for their comments, which improved our manuscript. This research is supported by the Air Force Research Laboratory under Grants DTRA01-01-C-0076 and FA8718-05-C-0021. Facility support from the W.M. Keck Foundation is also acknowledged. Contribution no. 482 of the CSIDE, IGPP, University of California, Santa Cruz.

\section{References}

Bonner, J. B., H. J. Patton, A. C. Rosca, H. Hooper, J. Orrey, M. Leidig, and I. Gupta (2003). Aspects of Rg and Lg generation from the Shagan depth of burial explosions, in Proc. of the 25th Seismic Research Review, Nuclear Explosion Monitoring: Building the Knowledge Base, Tucson, Arizona, 23-25 September 2003, 384-394.

Bottone, S., M. D. Fisk, and G. D. McCartor (2002). Regional seismic event 


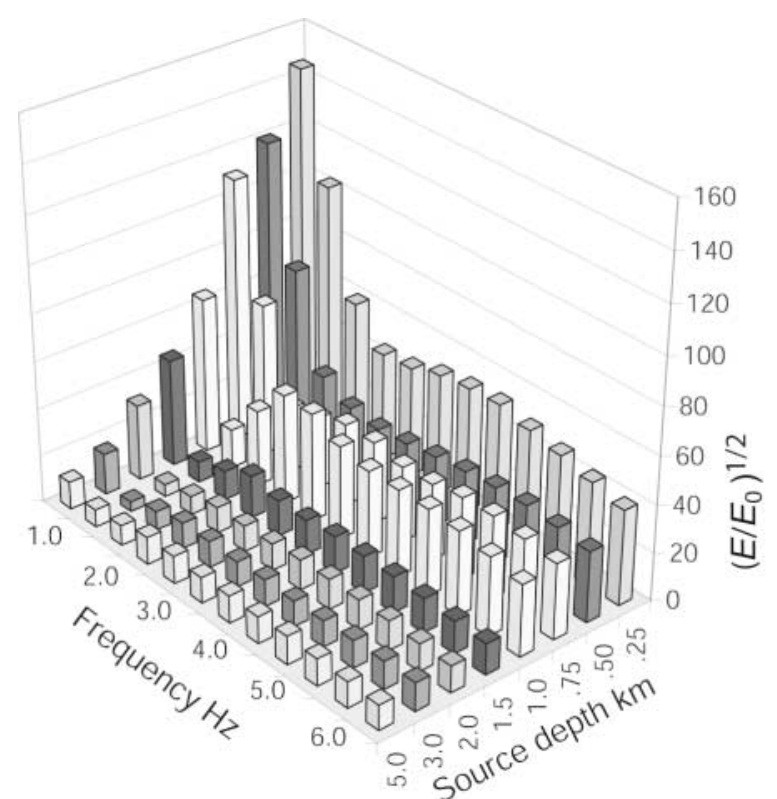

(a) EK+3\% deep random

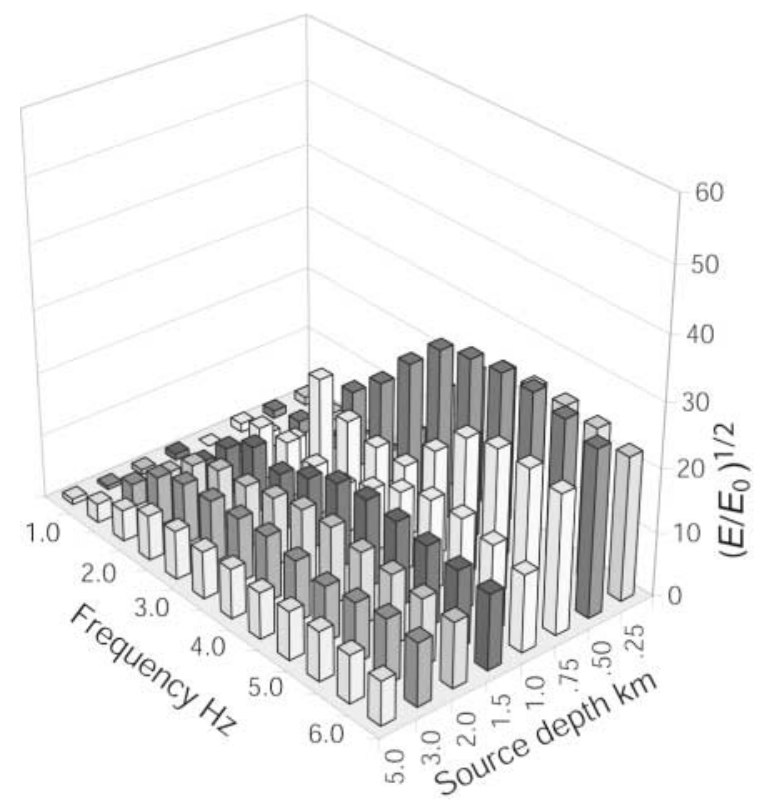

(c) $\mathrm{EK}+3 \%$ scattered energy

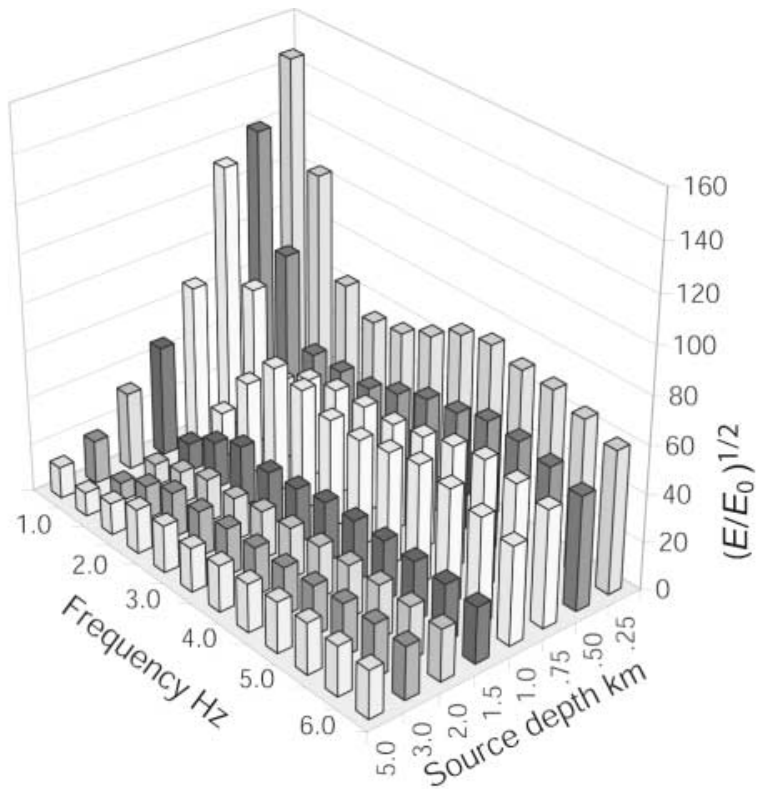

(b) EK $+5 \%$ deep random

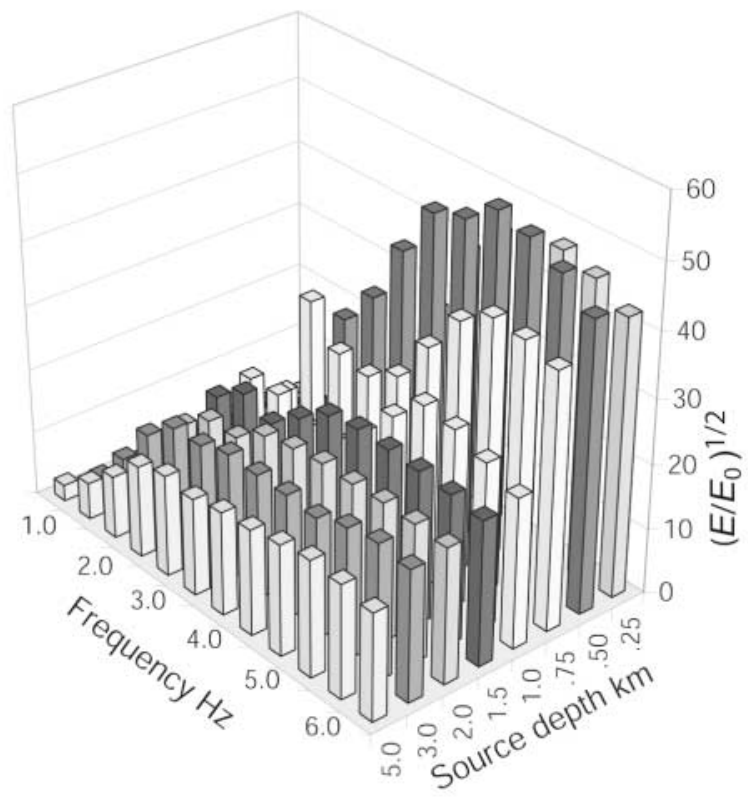

(d) EK $+5 \%$ scattered energy

Figure 16. Normalized $L g$-excitation spectra for sources in the EK model with deep random patches, for (a) the EK model with a 3\% deep random patch, (b) EK model with a $5 \%$ deep random patch, and (c) and (d) the isolated scattered energy in (a) and (b) due to the random patches.

characterization using a Bayesian formulation of simple kriging, Bull. Seism. Soc. Am. 92, 2277-2296.

Bradley, C. R., and E. M. Jones (1998). Modeling propagation effects from explosion in Western China and India, in Proc. of the 20th Annual Seismic Research Symposium on Monitoring a Comprehensive Nuclear-Test-Ban Treaty, Santa Fe, New Mexico, 21-23 September 1998, 173-181.

Bradley, C. R., and L. E. Jones (1999). Full waveform modeling of the effects of $\mathrm{Q}$ and structure over sub-regional paths, in Western China, in Proceedings of the 21st Annual Seismic Research Symposium on Monitoring a Comprehensive Nuclear-Test-Ban Treaty, Las Vegas, Nevada, 21-24 September 1999, 28-38.

Day, S. M., and K. L. McLaughlin (1991). Seismic source representations for spall, Bull. Seism. Soc. Am. 81, 191-201.

Fan, G. W., and T. Lay (1998a). Statistical analysis of irregular waveguide influences on regional seismic discriminants in China, Bull. Seism. Soc. Am. 88, 74-88.

Fan, G. W., and T. Lay (1998b). Regionalized versus single-station wave- 
guide effects on seismic discriminants in western China, Bull. Seism. Soc. Am. 88, 1260-1274.

Fan, G. W., and T. Lay (1998c). Statistical analysis of irregular waveguide influences on regional seismic discriminants in China: additional results for Pn/Sn, Pn/Lg and Pg/Sn, Bull. Seism. Soc. Am. 88, 15041510 .

Fisk, M. D., H. L. Gray, and G. D. McCartor (1996). Regional discrimination without transporting thresholds, Bull. Seism. Soc. Am. 86, $1545-1558$.

Frankel, A. (1989). A review of numerical experiments on seismic wave scattering, in Scattering and Attenuation of Seismic Waves, II, R. S. Wu and K. Aki (Editors), Birkhauser, Berlin, 639-686.

Gupta, I. N., W. Chan, and R. Wagner (1992). A comparison of regional phases from underground nuclear explosions at East Kazakh and Nevada Test Sites, Bull. Seism. Soc. Am. 82, 352-382.

Gupta, I. N., T. Zhang, and R. A. Wagner (1997). Low-frequency Lg from NTS and Kazakh nuclear explosions: observations and interpretations, Bull. Seism. Soc. Am. 87, 1115-1125.

Gutowski, P. R., F. Hron, D. E. Wagner, and S. Treitel (1984). S*, Bull. Seism. Soc. Am. 74, 61-78.

Hartse, H. E., S. R. Taylor, W. S. Phillips, and G. E. Randall (1997). A preliminary study of regional seismic discrimination in central Asia with emphasis on Western China, Bull. Seism. Soc. Am. 87, 551-568.

Jih, R. S. (1995). Numerical investigation of relative contribution of $\mathrm{Rg}$ scattering and incomplete dissipation to Lg excitation, in Proc. of the 17th Annual Seismic Research Symposium on Monitoring a Comprehensive Nuclear-Test-Ban Treaty, Scottsdale, Arizona, 12-15 September 1995, 401-410.

Jih, R. S. (1996). Waveguide effects of large-scale structural variation, anelastic attenuation, and random heterogeneity on SV Lg propagation: a finite-difference modeling study, in Proc. of the 18th Annual Seismic Research Symposium on Monitoring a Comprehensive Nuclear-TestBan Treaty, Annapolis, Maryland, 4-6 September 1996, 182-194.

Johnson, L. R., and C. G. Sammis (2001). Effects of rock damage on seismic waves generated by explosions, Pageoph 158, 1869-1908.

Kennett, B. L. N. (1989). Lg-wave propagation in heterogeneous media, Bull. Seism. Soc. Am. 79, 860-872.

Kennett, B. L. N., and S. Mykkeltveit (1984). Guided wave propagation in laterally varying media, II. Lg wave in northwestern Europe, Geophys. J. R. Astr. Soc. 79, 257-267.

Kim, W. Y., V. Aharonian, A. L. Lerner-Lam, and P. G. Richards (1997). Discrimination of earthquakes and explosions in southern Russia using regional high-frequency three-component data from IRIS/JSP Caucasus Network, Bull. Seism. Soc. Am. 87, 569-588.

Kim, W. Y., D. W. Simpson, and P. G. Richards (1993). Discrimination of earthquakes and explosions in the eastern United States using regional high-frequency data, Geophys. Res. Lett. 20, 1507-1510.

Lilwall, R. C. (1988). Regional $\mathrm{m}_{\mathrm{b}}: \mathrm{M}_{\mathrm{s}}, \mathrm{Lg} / \mathrm{Pg}$ amplitude ratios and $\mathrm{Lg}$ spectral ratios as criteria for distinguishing between earthquakes and explosions: a theoretical study, Geophys. J. Int. 93, 137-147.

Myers, S. C., J. Wagoner, S. Larsen, A. Rodgers, K. Mayeda, K. Smith, and W. Walter (2003). Simulation of regional explosion S-phases (SIRES) project, in Proc. of the 25th Seismic Research Review, Nuclear Explosion Monitoring: Building the Knowledge Base, Tucson, Arizona, 23-25 September 2003, 117-124.

Nuttli, O. W. (1986). Yield estimates of Nevada Test Site explosions obtained from Lg waves, J. Geophys. Res. 91, 2137-2151.

Priestley, K. F., G. Zandt, and G. E. Randall (1988). Crustal structure in
Eastern Kazakh, USSR from teleseismic receiver functions, Geophys. Res. Lett. 15, 613-616.

Patton, H. J. (2001). Regional magnitude scaling, transportability, and Ms:mb discrimination at small magnitudes, Pageoph 158, 19512015.

Sato, H., and M. C. Fehler (1998). Seismic Wave Propagation and Scattering in the Heterogeneous Earth, Springer-Verlag, New York.

Stevens, J. L., G. E. Baker, H. Xu, T. J. Bennett, N. Rimer, and S. M. Day (2003). The physical basis of Lg generation by explosion sources, in Proc. of the 25th Seismic Research Review, Nuclear Explosion Monitoring: Building the Knowledge Base, Tucson, Arizona, 23-25 September 2003, 456-465.

Taylor, S. R. (1996). Analysis of high frequency Pn/Lg ratios from NTS explosions and western U.S. earthquakes, Bull. Seism. Soc. Am. 86, 1042-1053.

Taylor, S. R., M. D. Denny, E. S. Vergino, and R. E. Glaser (1989). Regional discrimination between NTS explosions and western U.S. earthquakes, Bull. Seism. Soc. Am. 79, 1142-1176.

Taylor, S. R., and H. E. Hartse (1997). An evaluation of generalized likelihood ratio outlier detection to identification of seismic events in Western China, Bull. Seism. Soc. Am. 87, 824-831.

Vogfjord, K. S. (1997). Effects of explosion depth and earth structure on the excitation of $\mathrm{Lg}$ waves: $\mathrm{S}^{*}$ revisited, Bull. Seism. Soc. Am. 87, 1100-1114.

Wallace, T. C. (1991). Body wave observations of tectonic release, in $E x$ plosion Source Phenomenology, S. R. Taylor, H. J. Patton, and P. G. Richards (Editors), American Geophysical Union, Washington, D.C., 161-170.

Walter, W. R., K. M. Mayeda, and H. Patton (1995). Phase and spectral ratio discrimination between NTS earthquakes and explosions, part I: empirical observations, Bull. Seism. Soc. Am. 85, 1050-1067.

Wu, R. S., and K. Aki (Editors) (1988). Scattering and Attenuation of Seismic Waves, Vol. I, Birkhauser Verlag, Boston.

Wu, R. S., S. Jin, and X. B. Xie (2000a). Seismic wave propagation and scattering in heterogeneous crustal waveguides using screen propagators: I SH waves, Bull. Seism. Soc. Am. 90, 401-413.

Wu, R. S., S. Jin, and X. B. Xie (2000b). Energy partition and attenuation of $\mathrm{Lg}$ waves by numerical simulations using screen propagators, $P$ hys. Earth Planet. Interiors 120, 227-243.

Xie, J. (2002). Source scaling of Pn and Lg spectra and their ratios from explosions in central Asia: Implications for the identification of small seismic events at regional distances, J. Geophys. Res. 107, no. B7, 10.1029/2001JB000509.

Xie, J., L. L. Cong, and B. J. Mitchell (1996). Spectral characteristics of the excitation and propagation of $\mathrm{Lg}$ from underground nuclear explosions in central Asia, J. Geophys. Res. 101, 5813-5822.

Xie, X. B., and T. Lay (1994). The excitation of Lg waves by explosions: A finite-difference investigation, Bull. Seism. Soc. Am. 84, 324-342.

Institute of Geophysics and Planetary Physics

University of California

1156 High Street

Santa Cruz, California 95064

Manuscript received 7 February 2005. 